\title{
Individual alpha frequency modulates sleep-related emotional memory consolidation
}

\author{
Zachariah R. Cross ${ }^{\text {a, *, Amanda Santamaria }}{ }^{\text {a }, \text { Andrew W. Corcoran }}{ }^{\text {a,b }}$, Alex Chatburn ${ }^{\text {a, }}$ \\ Phillip M. Alday ${ }^{c}$, Scott Coussens ${ }^{\text {a }}$, Mark J. Kohler ${ }^{\text {a,d }}$ \\ ${ }^{a}$ Cognitive and Systems Neuroscience Research Hub, University of South Australia, Adelaide, Australia \\ ${ }^{\mathrm{b}}$ Cognition and Philosophy Laboratory, Monash University, Melbourne, Australia \\ ${ }^{\mathrm{c}}$ Max Planck Institute for Psycholinguistics, Nijmegen, 6500 AH, the Netherlands \\ ${ }^{\mathrm{d}}$ School of Psychology, Adelaide University, Adelaide, Australia
}

\section{A R T I C L E I N F O}

\section{Keywords:}

Individual alpha frequency

Sleep

Memory consolidation

Neuronal oscillations

Emotional memory

\begin{abstract}
A B S T R A C T
Alpha-band oscillatory activity is involved in modulating memory and attention. However, few studies have investigated individual differences in oscillatory activity during the encoding of emotional memory, particularly in sleep paradigms where sleep is thought to play an active role in memory consolidation. The current study aimed to address the question of whether individual alpha frequency (IAF) modulates the consolidation of declarative memory across periods of sleep and wake. 22 participants aged 18-41 years (mean age $=25.77$ ) viewed 120 emotionally valenced images (positive, negative, neutral) and completed a baseline memory task before a $2 \mathrm{hr}$ afternoon sleep opportunity and an equivalent period of wake. Following the sleep and wake conditions, participants were required to distinguish between 120 learned (target) images and 120 new (distractor) images. This method allowed us to delineate the role of different oscillatory components of sleep and wake states in the emotional modulation of memory. Linear mixed-effects models revealed interactions between IAF, rapid eye movement sleep theta power, and slow-wave sleep slow oscillatory density on memory outcomes. These results highlight the importance of individual factors in the EEG in modulating oscillatory-related memory consolidation and subsequent behavioural outcomes and test predictions proposed by models of sleep-based memory consolidation.
\end{abstract}

\section{Introduction}

The neural basis of sleep-associated memory consolidation has garnered significant attention within cognitive neuroscience (for review, see Rasch and Born, 2013), with it now being well established that sleep benefits hippocampus-dependent (i.e., declarative) memory (Ellenbogen et al., 2006; Klinzing et al., 2016). However, despite the large body of literature detailing the mechanisms underpinning the role of sleep in memory, only a small portion of studies have investigated individual differences in encoding and sleep-related memory processing in humans (e.g., Fenn and Hambrick, 2012; Schabus et al., 2008; Wislowska et al., 2017). One promising individual trait marker of cognitive function is the individual alpha frequency (IAF), often parameterised as the maximum (i.e. peak) power value within the alpha band $(\sim 8-13 \mathrm{~Hz})$ during resting-state measurement (Bazanova and Vernon, 2014; Grandy et al., 2013a; Klimesch, 1999). While IAF is known to differ among individuals and is correlated with memory and general intelligence (Klimesch, 1999), the relation between encodingand sleep-related memory processing and IAF remains unknown. Here, we aim to determine whether IAF - as a proxy of inter-individual differences in information processing - interacts with oscillatory mechanisms of sleep-associated memory consolidation to influence human declarative memory. We also examine whether the emotional valence of to-be-learned information interacts with IAF to influence behavioural outcomes after sleep, given that emotional information is often prioritised over neutral stimuli during encoding (Murty et al., 2010) and sleep-based memory processing (Bennion et al., 2015; Sterpenich et al., 2009).

\subsection{Sleep-related memory consolidation}

During sleep, memory processing involves the replay of neural firing

\footnotetext{
* Corresponding author.

E-mail addresses: zachariah.cross@unisa.edu.au, zachariah.cross@mymail.unisa.edu.au (Z.R. Cross).
} 
patterns present during encoding, with this reactivation possibly reflecting the transmission of information from the hippocampal complex to neocortex for long-term storage (Hanert et al., 2017; Helfrich et al., 2019). Such hippocampal-cortical communication, as posited by the Active System Consolidation model (Born and Wilhelm, 2012; Rasch and Born, 2013), is facilitated by three cardinal oscillatory rhythms during slow wave sleep (SWS): hippocampal sharp-wave ripples $(\sim 200$ $\mathrm{Hz})$, which are nested within thalamic spindles $(\sim 12-16 \mathrm{~Hz})$, which are in turn nested in the up-state of neocortically generated slow oscillations (SOs; 0.5-1 Hz; Helfrich et al., 2018; Staresina et al., 2015). In addition to supporting gist abstraction (Chatburn et al., 2014), rule generalisation (Batterink et al., 2014; Cross et al., 2020; Lutz et al., 2018) and in transforming episodic memory traces (Inostroza and Born, 2013), sleep has also been shown to preferentially consolidate emotional over neutral information (Diekelmann et al., 2009; Groch et al., 2013; Hutchison and Rathore, 2015; Payne et al., 2015); however, a recent meta-analysis (Lipinska et al., 2019) found mixed results, with sleep also benefiting the consolidation of neutral over emotional information (e.g., Cellini et al., 2016).

Of the physiological processes active during sleep, rapid eye movement sleep (REM) has been implicated in emotional memory consolidation (cf. Morgenthaler et al., 2014), particularly via REM theta oscillations, which are proposed to represent homeostatic processes of emotional brain regulation and have been linked to the synchronisation of emotional information between the amygdala and hippocampus (Groch et al., 2013; Wagner et al., 2001; Diekelmann et al., 2009; Hutchison and Rathore, 2015; Prehn-Kristensen et al., 2013). However, REM neurophysiology is unlikely to solely explain sleep-related emotional memory consolidation (Morgenthaler et al., 2014). Alternatively, according to the Synaptic Homeostasis Hypothesis (Tononi and Cirelli, 2014), SOs during SWS may facilitate the consolidation of emotional information through decreasing synaptic connectivity and selectively refining emotional over neutral memory representations, while spindles serve to promote memory consolidation via synaptic long-term potentiation (Morgenthaler et al., 2014; Payne et al., 2015; Walker, 2010). As such, it is possible that both SWS oscillatory activity (e.g., SOs and spindles) and REM theta oscillations are important for the consolidation of emotional memory (Cunningham et al., 2014; Hutchison and Rathore, 2015).

Evidence also indicates that shorter sleep durations, such as an afternoon nap, are also effective in stabilising new information (e.g., Nishida et al., 2009; Payne et al., 2015). An afternoon nap occurs at a different circadian phase than nocturnal sleep and is typically dominated by non-rapid eye movement (NREM) sleep (Payne et al., 2015). Afternoon naps also allow relatively better control of potential time-of-day effects on performance, and if sleep facilitates long-term memory through a process of active consolidation, then emotional memory should benefit from an afternoon nap compared to an equivalent period of wake. However, while the SWS and REM oscillatory mechanisms underpinning the emotional modulation of memory are beginning to be well characterised (e.g., Cairney et al., 2014; Groch et al., 2015; Hutchison and Rathore, 2015), far less is known regarding individual differences in information processing and cognitive ability and their influence on sleep-associated memory consolidation.

\subsection{Individual differences in sleep-related memory consolidation}

Of the studies examining individual differences in sleep and memory, baseline memory performance (Wislowska et al., 2017), general intelligence ( $g$; Fenn and Hambrick, 2015), working memory capacity (Fenn and Hambrick, 2012) and interindividual sleep spindle differences (Schabus et al., 2008) have been shown to modulate memory change over sleep relative to wake. For example, a higher working memory capacity is predictive of greater memory retention across sleep (Fenn and Hambrick, 2012), suggesting that beneficial effects of sleep on memory may partially rely on individual differences in the formation and maintenance of stimulus representations during online information processing. Further, individuals with higher $g$ show greater memory gains across sleep (Fenn and Hambrick, 2015), indicating that interindividual differences in $g$ may reflect online and sleep-related cognitive processing capacities. It should also be noted that sleep spindles have been related to broad cognitive functioning (for review, see Fernandez and Luthi, 2020, \& Ujma, 2018), including general intelligence (Fogel and Smith, 2011), and the development of sensory-motor functioning and working memory in children (Chatburn et al., 2013). However, while these studies have provided important initial insights into the interindividual factors modulating sleep and memory, little is known regarding the neurobiological factors underpinning such interindividual differences, particularly during online information processing.

One potential neurobiological candidate reflecting interindividual differences in information processing and cognition is IAF, the predominant frequency of alpha-band oscillations $(\sim 8-13 \mathrm{~Hz}$; Klimesch, 1999). Research reveals that IAF is predictive of both perceptual (Cecere et al., 2015; Samaha and Postle, 2015) and higher-order cognitive task performance (Bornkessel et al., 2004; Bornkessel-Schlesewsky et al., 2015; Klimesch et al., 2006), with low IAF individuals showing slower processing speeds (Klimesch et al., 1996), and lower performance on both memory tasks (Klimesch, 1999; Klimesch et al., 1993) and measures of general intelligence (Grandy et al., 2013a). IAF is also highly heritable (Posthuma et al., 2001), demonstrates high test-retest reliability and declines with age (Grandy et al., 2013b), partially explaining age-related reductions in cognition over the lifespan (e.g., Bornkessel-Schlesewsky et al., 2015). From this perspective, IAF may serve as a useful proxy for general cognitive and brain functioning, including individual differences in both working and long-term memory and measures of general intelligence; however, its relation to sleep-associated memory consolidation remains virtually unknown.

Although IAF has yet to be explicitly related to sleep oscillatory activity, there are numerous sources of evidence which would suggest this is a reasonable belief to hold. As IAF is positively related to (working) memory performance and general intelligence measures, both of which modulate sleep-associated memory consolidation, IAF may also modulate memory encoding and subsequent consolidation during sleep. Further, neurophysiological evidence has linked wake alpha oscillations with sleep-based spindle oscillations (for full discussions, see Cox et al., 2017; Bazanova and Vernon, 2014; see also Lechinger et al., 2015). If sleep plays an active role in memory consolidation through specific patterns of oscillatory activity (i.e., thalamic spindles, SOs, REM theta activity), and if IAF modulates online information processing (e.g., including attention and perception), IAF should further modulate memory performance by interacting with sleep-based oscillatory activity.

IAF is considered a marker of attentional control, with higher-IAF individuals exhibiting better attentional gating control (i.e., a better ability to filter irrelevant stimuli and inhibit task-irrelevant cortical networks; Klimesch, 2012). As such, IAF may also modulate interindividual differences in emotional memory by reflecting attentional discriminatory mechanisms that prioritise emotional over neutral information at encoding and during sleep-associated memory consolidation (e.g., Saletin and Walker, 2012). Specifically, emotional information attracts more elaborative encoding strategies, such as autobiographical and semantic elaboration, as well as selective enhancement of encoding processes through arousal activation (Crowley et al., 2019) and pre-activation of task-relevant networks (Jann et al., 2010). The resulting induced facilitation of emotional item encoding leads to improved consolidation via activation of the basolateral amygdala and medial temporal lobe (LeDoux, 2007; McGaugh, 2004), enabling stronger sleep-related memory consolidation of emotional over neutral information. However, the relation between IAF and emotional modulation of information across sleep has not been studied. 


\subsection{The current study}

The present study aimed to determine whether IAF facilitates sleeprelated memory consolidation by interacting with sleep neural oscillatory mechanisms and whether this facilitation is higher for emotional compared to neutral stimuli. To this end, participants completed a memory task involving images of varying valence. Immediate and delayed recall tasks were completed to assess memory before and after an intervening $2 \mathrm{hr}$ afternoon nap and equivalent period of wake. We recorded electroencephalography (EEG) during eyes open and closed resting-state periods to estimated participants' IAF values. EEG was also recorded continuously during the sleep period, with which we quantified theta spectral activity $(\sim 4-7 \mathrm{~Hz})$ during REM. Using an automated detection algorithm, we also estimated spindle and SO density during SWS and examined the relationship between IAF, emotional valence and sleep EEG oscillatory mechanisms on memory performance using linear mixed-effects modelling.

It was hypothesised that condition (sleep, wake), IAF and emotional valence (positive, negative, neutral) would interact in their effect on emotional memory performance. Specifically, it was predicted that memory performance (d' scores) would be greater after sleep compared to wake $\left(\mathrm{H}^{1}\right)$, and that this effect would be further accentuated for emotional compared to neutral information $\left(\mathrm{H}^{2}\right)$. Further, we sought to examine whether IAF and sleep physiology interact to influence memory.

\section{Method}

\subsection{Participants}

Participants included 22 right-handed healthy adults (10 male) ranging from 18 to 41 years old (mean age $=25.77$ ). A power analyses using G*Power 3 (Faul et al., 2007) of a previous study examining the impact of sleep on emotional memory (partial $\eta^{2}=0.20$ based on Payne et al., 2008) suggested a sample size of 12 would be adequate to detect similar sized effects in a repeated measures design $(1-\beta=0.80, \alpha=.05)$. All participants reported normal or corrected-to-normal vision and hearing and had no current or past psychiatric conditions, substance dependence or abuse, intellectual impairment and were not taking medication that influenced sleep and neuropsychological measures. One participant failed to return for the second session, resulting in a final sample size of 21 ( 9 male; mean age $=25.95$ ). All participants provided informed consent and received a AUD $\$ 40$ honorarium. Ethics for this study was granted by the University of South Australia's Human Research Ethics committee (I.D: 0000032556).

\subsection{Design}

This study was a repeated measures within-subjects experimental design with two conditions (sleep, wake). Each condition was counterbalanced across participants and separated by one week to control for condition order effects and to avoid interference between task sets. Conditions included:

a) Sleep condition: Participants underwent learning with an immediate retrieval task followed by a $2 \mathrm{hr}$ sleep opportunity. A delayed retrieval task occurred 30 min after waking.

b) Wake condition: Participants underwent learning with an immediate retrieval task. This was followed by a delayed retrieval task after a $2 \mathrm{hr}$ wake period.

\subsection{Materials and measures}

\subsubsection{Demographic measures}

Participants completed a paper questionnaire containing questions on age, sex, ethnicity, highest level of education achieved and recent
$(<24 \mathrm{hr}$ ) alcohol and caffeine consumption, as caffeine and alcohol are known to influence performance on cognitive tasks (e.g., Keenan et al., 2014). All participants reported no consumption of alcohol the previous evening or caffeine on the day of testing.

\subsubsection{Screening and control measures}

The Pittsburgh Sleep Quality Index (PSQI; Buysse et al., 1989) was used to screen for sleep quality. Participants' PSQI scores ranged from 1 to $5(M=3.4, S D=1.50)$, indicating good sleep quality. The Weschler Abbreviated Scale of Intelligence (WASI-II) was used to index intellectual ability, as intelligence may influence memory retention and performance on memory tasks (Conway et al., 2003), and is correlated with IAF (Grandy et al., 2013a). The WASI-II provides an estimate of full-scale IQ (FSIQ). Participants' mean FSQI score was 114 (SD = 16.44), placing participants in a high range of intellectual functioning (Wechsler, 2011). The visual analogue scale for sleepiness (VASS) was also used to index sleepiness. The VASS is comprised of a $100 \mathrm{~mm}$ scale with "sleepy/drowsy" and "alert/awake" for endpoints to denote a continuum of state sleepiness. Participants indicated where on the line they judged their current state of sleepiness prior to the start and end of each learning and recall session. Scores were determined as mm distance from the left pole to the participants' mark, indicating degree of sleepiness as a percentage, with lower scores indicating greater sleepiness.

\subsubsection{Polysomnography (PSG)}

PSG was recorded using the Compumedics Grael High-Definition PSG 24-bit amplifier (Compumedics Pty Ltd., Melbourne, Australia). Electrodes were arranged according to the International 10-20 System (American Electroencephalographic Society, 1994) at the following locations: FP1, FP2, F3, F4, C3, C4, T7, T8, P3, P4, P7, P8, O1, O2. In addition to left and right electro-oculography (EOG), sub-mental electromyography (EMG) and electrocardiography (ECG), EEG was recorded and referenced to contralateral mastoids and sampled at a rate of $1064 \mathrm{~Hz}$ with a bandpass filter from DC to $143 \mathrm{~Hz}$. All impedances were kept at or below $10 \mathrm{k} \Omega$ throughout recording periods. All sleep data were scored by an experienced sleep technician according to standardised criteria (Berry et al., 2012) with EEG viewed with a high pass filter of $0.3 \mathrm{~Hz}$ and a low pass filter of $35 \mathrm{~Hz}$. The following sleep parameters were derived from PSG recordings: time in bed, total sleep time (TST), sleep onset latency (SOL; time from lights out to the first epoch of sleep), REM onset latency, sleep efficiency (SE) [(total sleep time/time in bed) $\mathrm{x}$ 100], wake after sleep onset, total arousal index, duration and percent of TST spent in each sleep stage.

\subsection{Emotional memory task}

480 pictures were used from the International Affective Picture System (IAPS; Lang et al., 2008), which were divided into two parallel sets, counterbalanced across the Sleep and Wake conditions. The IAPS are rated for emotionality based on two dimensions (valence: $1=$ unpleasant, $9=$ pleasant; arousal: $1=$ calm, $9=$ excited) by a normative adult sample (Lang et al., 2008). Mean valence and arousal ratings of the stimuli have high internal consistency $(a=0.94)$ and split-half reliability ( $r s=0.94)$.

Stimuli were displayed via OpenSesame v.2.9.7 (Mathôt et al., 2012) and were categorised into three groups (i.e., negative, neutral, positive) according to their mean valence values. All stimuli were counterbalanced based on arousal. In accordance with recommendations by Sassenhagen and Alday (2016), means and standard deviations for valence and arousal values per stimulus category (positive, negative, neutral) and set (A, B) were generated to ensure that they were not overlapping (see Table 1 for the descriptive statistics of the stimuli used in Set A and Set B). As is clear from Fig. 1A, the valence values for negative, neutral and positive stimuli are largely non-overlapping, indicating that negative stimuli were more negatively valenced than neutral and positive stimuli, and that positive stimuli were more 
Table 1

Means and standard deviations of the valence (1, negative to 9, positive) and arousal (1, calming to 9, highly arousing) values of the learning and recall stimuli in Set A and Set B.

\begin{tabular}{|c|c|c|c|c|c|c|}
\hline & \multicolumn{3}{|l|}{ Set A } & \multicolumn{3}{|l|}{ Set B } \\
\hline & Negative & Neutral & Positive & Negative & Neutral & Positive \\
\hline \multicolumn{7}{|l|}{ Learning } \\
\hline \multirow[t]{2}{*}{ Valence } & 3.28 & 5.01 & 6.94 & 3.31 & 5.04 & 7.03 \\
\hline & $(.67)$ & $(.26)$ & $(.62)$ & $(.65)$ & $(.26)$ & $(.65)$ \\
\hline \multirow[t]{2}{*}{ Arousal } & 5.24 & 3.41 & 4.70 & 5.25 & 3.51 & 4.68 \\
\hline & $(.87)$ & $(.80)$ & (1.05) & $(.82)$ & $(.85)$ & (1.05) \\
\hline \multicolumn{7}{|l|}{ Recall } \\
\hline \multirow[t]{2}{*}{ Valence } & 3.22 & 4.98 & 7.00 & 3.29 & 5.05 & 7.00 \\
\hline & $(.71)$ & $(.25)$ & $(.65)$ & $(.67)$ & $(.24)$ & $(.67)$ \\
\hline \multirow[t]{2}{*}{ Arousal } & 5.21 & 3.44 & 4.70 & 5.28 & 3.53 & 4.70 \\
\hline & $(.87)$ & $(.78)$ & $(1.00)$ & $(.94)$ & $(.93)$ & (1.03) \\
\hline
\end{tabular}

Note. Standard deviations are in parentheses. Valence and arousal scores were derived from the 9-point version of the self-assessment manikin (Lang et al., 2010). positively valenced than neutral and negative stimuli. Further, Fig. 1B indicates that positive and negative stimuli were more arousing than neutral stimuli, and that positive and negative stimuli arousal values were overlapping.

During the learning tasks, 120 stimuli for each set, each containing 40 neutral, 40 positive and 40 negatively valenced images, were presented sequentially for participants to learn, followed by an immediate retrieval task to gain a recognition baseline. During the learning phase, participants were instructed to attend to each image and to memorise it for later testing. The delayed retrieval task occurred post experimental conditions. The immediate and delayed retrieval tasks contained the 120 target pictures shown during learning intermixed with the 120 distractor pictures shown during the immediate retrieval task (see Fig. 2 for a schematic representation). Following presentation, participants judged whether pictures were targets (old; i.e., seen during learning) or distractors (new; i.e., not seen during learning). Pictures were pseudorandomised at each time of testing, such that no more than two pictures of the same emotion followed. Testing time for each experimental task was approximately $20-30 \mathrm{~min}$.
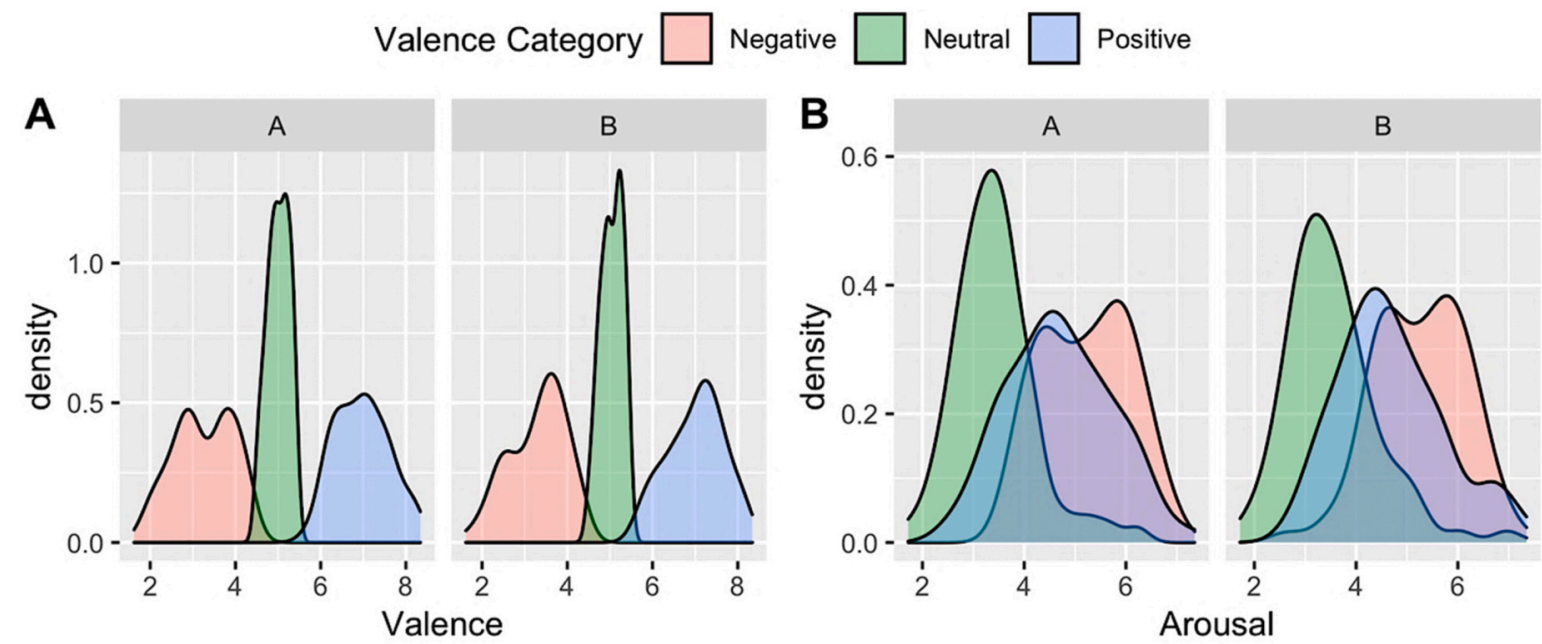

Fig. 1. Density plots illustrating the difference in the distribution of valence (A) and arousal (B) values for each valence category (negative, neutral, positive) and stimulus set (A, B). Higher scores on Figure A indicate more positive valence values, while lower scores indicate more negative valence values. Higher scores on Figure B indicate more arousing values, while lower scores indicate less arousing values.

(A)

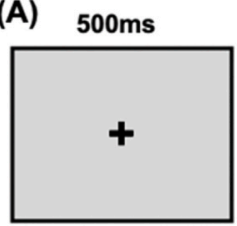

(B)

(B) $500 \mathrm{~ms}$

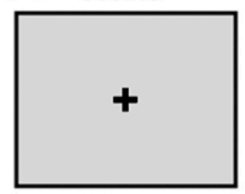

$1000 \mathrm{~ms}$

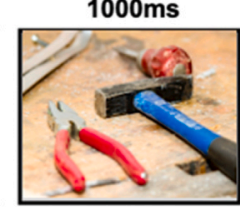

$1000 \mathrm{~ms}$

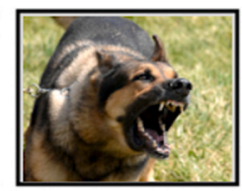

$500 \mathrm{~ms}$

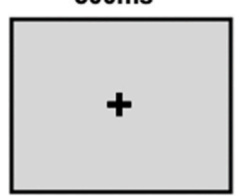

$5000 \mathrm{~ms}$

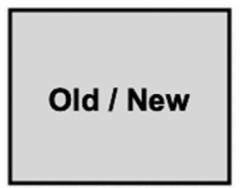

$1000 \mathrm{~ms}$

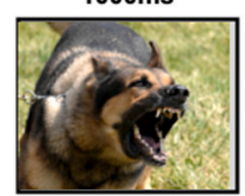

$500 \mathrm{~ms}$

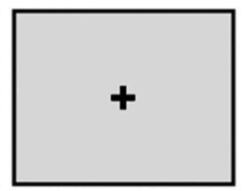

$500 \mathrm{~ms}$

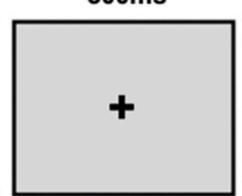

$1000 \mathrm{~ms}$

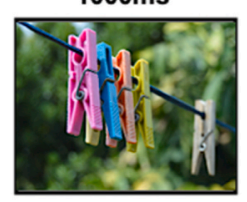

$1000 \mathrm{~ms}$

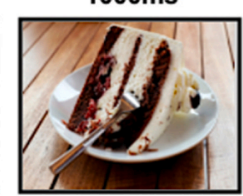

$5000 \mathrm{~ms}$

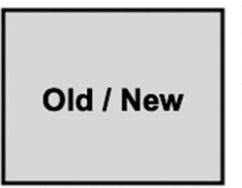

$500 \mathrm{~ms}$

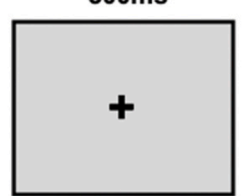

$500 \mathrm{~ms}$

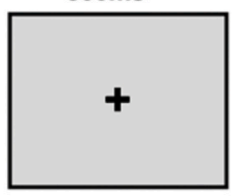

Time (ms)

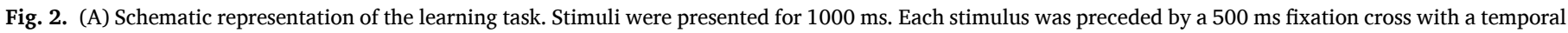

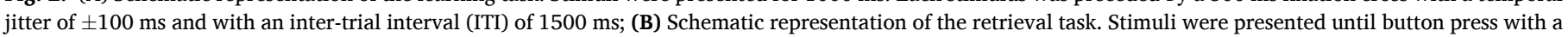

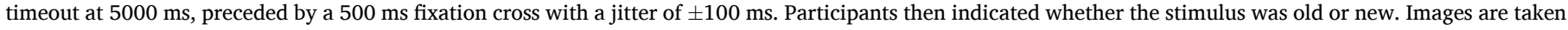
from Creative Commons for illustrative purposes, as the IAPS are restrictively licensed and not available for general distribution. 


\subsection{Procedure}

Participants attended the laboratory at approximately 11:45 h and were taken through to the testing rooms for PSG/EEG set-up. Resting state EEG activity was recorded during quiet sitting with eyes open (focussing on a fixation cross centred on a computer monitor) and eyes closed for $2 \mathrm{~min}$, respectively. Participants then completed the learning task by approximately $13: 30 \mathrm{~h}$, followed by the immediate recall task.

During the wake condition, participants remained in the laboratory and were administered the WASI-II and were permitted to engage in non-strenuous activity (e.g., reading, talking between other participants and experimenters, drawing). During the sleep condition, participants were given a 120-min sleep opportunity between the hours of $14: 30 \mathrm{~h}$ and $16.30 \mathrm{~h}$. Nap success rate was $100 \%$ (defined as obtaining at least 10 min of sleep, per Alger et al., 2012). PSG data were used to verify sleep periods. Participants engaged in non-strenuous activity for $30 \mathrm{~min}$ after the nap until testing to alleviate inertia effects on memory performance (Cairney et al., 2014; Payne et al., 2008; Tassi and Muzet, 2000). At approximately 17:00 h, participants completed the delayed retrieval task (see Fig. 3 for study protocol).

\section{Data analysis}

\subsection{Behavioural data}

Memory performance was calculated based on signal detection theory (Stanislaw and Todorov, 1999). Hit Rate (HR) and False Alarm rate (FA) were computed to derive the discrimination index (d'), defined as the difference between the $\mathrm{z}$ transformed probabilities of HR and FA (i. e., d' $=\mathrm{z}[\mathrm{HR}]-\mathrm{z}[\mathrm{FA}]$ ). Adjustment of extreme values (i.e., HR and FA values of 0 and 1) was made using the recommendations of Hautus (1995). Difference scores were calculated by subtracting Immediate testing d' scores from Delayed testing d' scores in order to estimate changes in memory retention across the sleep and wake periods.

\subsection{EEG pre-processing and analysis}

\subsubsection{Individual alpha frequency estimation}

Resting-state EEG pre-processing was conducted using customised scripts programmed in MATLAB ${ }^{\circ}$ (R2015a, The MathWorks, Inc., Natick, MA, USA). EOG and parieto-occipital EEG channels (P3-M2, P4$\mathrm{M} 1, \mathrm{O} 1-\mathrm{M} 2$, and O2-M1) were imported into MATLAB via the EEGLAB toolbox (v.13.6.5b; Delorme and Makeig, 2004) and subjected to zero-phase, finite impulse response highpass (passband edge: $1 \mathrm{~Hz},-6$ dB cutoff: $0.5 \mathrm{~Hz}$ ) and lowpass (passband edge: $40 \mathrm{~Hz},-6 \mathrm{~dB}$ cutoff: 45 $\mathrm{Hz}$ ) Hamming-windowed sinc filters (implemented via the pop_eegfiltnew function of the firfilt plugin; v1.6.1). An automated artifact detection routine (implemented via the pop_continuousartdet function in ERPLAB; Lopez-Calderon and Luck, 2014) was applied in order to exclude blinks and other sources of signal contamination. The peak-to-peak threshold for artifact rejection was set at $\pm 75 \mu \mathrm{V}$, and applied within a $500 \mathrm{~ms}$ sliding window ( $50 \%$ overlap). EOG channels were removed from the data following artifact rejection.

IAF estimates were obtained using restingIAF v1.0.3 (Corcoran et al., 2019), an open-source package available from https://github.com/corc orana/restingIAF. This automated IAF estimation routine uses a Savitzky-Golay filter (frame length $=11$ frequency bins, polynomial degree $=5$ ) to smooth the power spectral density (PSD). It then searches the first derivative of the smoothed PSD for evidence of peak activity within a defined frequency interval (here, 7-13 Hz). Given the low number of parieto-occipital channels available for analysis (i.e., 4), the minimum number of valid channel estimates required to estimate IAF was set to 1 . Peak frequencies were calculated from eyes-closed resting-state recordings (estimates from the Sleep and Wake conditions were grand-averaged, unless only one estimate was available). All other analysis parameters were per default settings (see Corcoran et al., 2018).

\subsubsection{Sleep EEG analyses}

Sleep EEG analyses were performed to examine the association between IAF and emotional memory consolidation with specific sleep oscillatory parameters during both SWS (i.e., spindles and SO density) and REM (i.e., theta power). Analyses were performed using the SpiSOP toolbox (https://www.spisop.org; RRID: SCR_015673), run in MATLAB 2016b (Mathworks, Natick, USA) and Fieldtrip (Oostenveld et al., 2011). Briefly, the EEG data were downsampled from $1064 \mathrm{~Hz}$ to $128 \mathrm{~Hz}$ to reduce computational intensity. PSD estimates were then calculated on consecutive $5 \mathrm{~s}$ intervals, which overlapped by $4 \mathrm{~s}$. Intervals were tapered by a single Hanning window before applying a fast Fourier transformation that resulted in interval power spectra with a frequency resolution of $0.2 \mathrm{~Hz}$. Power spectra were then averaged across all blocks (Welch's method) and normalised by the effective noise bandwidth to obtain PSD estimates for theta activity $(4-8 \mathrm{~Hz})$ during REM based on channel C3.

Spindle detection algorithms were based on Mölle et al. (2002) but were adapted to account for inter-individual differences in centre spindle frequencies, which is known to differ among individuals (e.g., Cox et al., 2017; Klimesch, 2018). Briefly, the EEG signal was band-pass filtered using a finite impulse response filter, which was defined based on individually estimated centre spindle frequencies. Power spectral density plots were generated to visualise peak spindle frequency estimates for each subject. From these, the peak spindle frequency for each subject was determined, which we then used to define the spindle

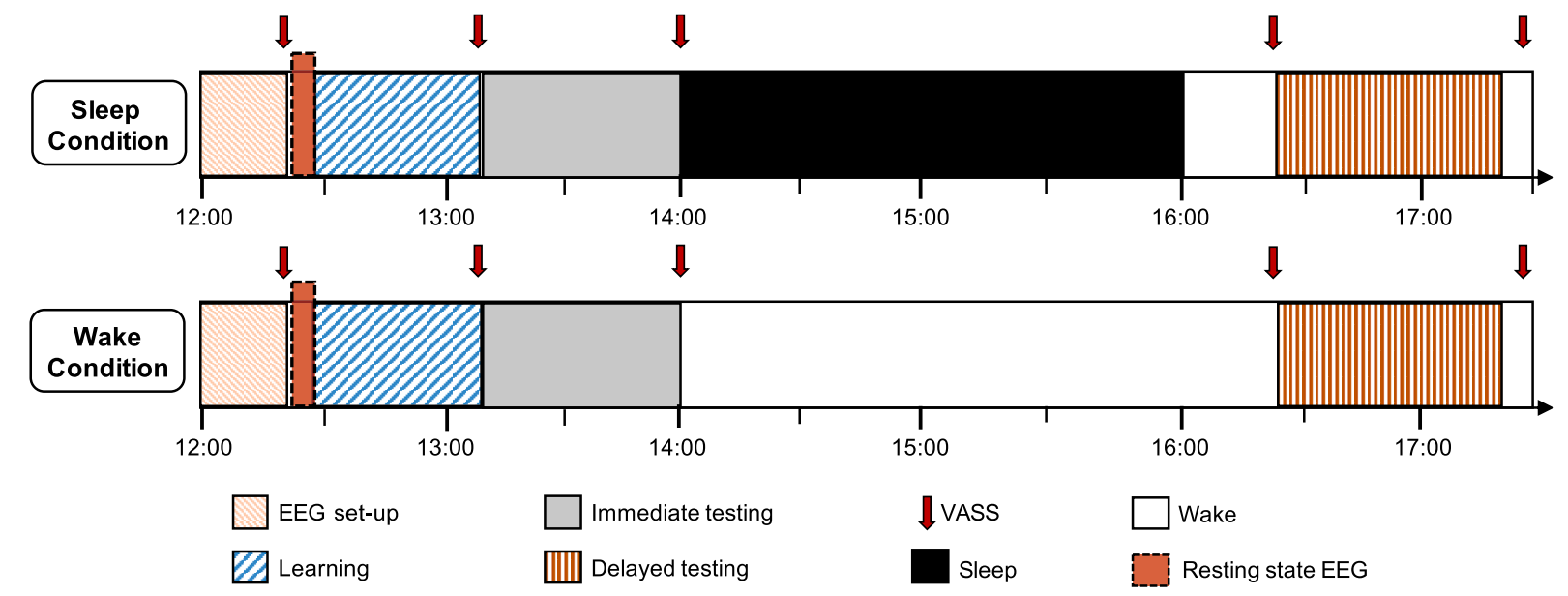

Fig. 3. Diagram representing the time course of the experimental conditions (sleep, wake) and testing session (learning, immediate and delayed retrieval). 
frequency range, defined as $\pm 2 \mathrm{~Hz}$ the centre frequency. This approach is more sensitive to individual differences in spindle-related analyses, rather than applying the canonical $12-16 \mathrm{~Hz}$ range to each subject (e.g., Helfrich et al., 2019). Then, using a sliding window with a size of $0.2 \mathrm{~s}$, the root mean square (RMS) was computed. The resulting signal was then smoothed in the same window with a moving average. A spindle was detected when the smoothed RMS signal exceeded an individual amplitude threshold 1.5 times the standard deviation of the filtered signal for $0.5-3 \mathrm{~s}$. Spindle density (i.e., $n$ spindles per $30 \mathrm{~s}$ SWS epoch) was then calculated for each subject at channel C3 (Carrier et al., 2001; Piosczyk et al., 2013; Schabus et al., 2004; Studte et al., 2017; van Schalkwijk et al., 2019).

Finally, SO detection was based on Mölle et al. (2002; also see Ngo et al., 2013). Briefly, the EEG signal was high and low pass filtered to isolate frequency components in the SO band (i.e., 0.3-3.5 Hz). All events with consecutive positive-to-negative zero crossings were then flagged, with components with durations corresponding to a minimum and maximum SO frequency considered as putative SOs, as per Mölle et al. (2002). Slow oscillation density (i.e., $n$ SO events per 30 s SWS epoch) was then calculated per subject.

\subsection{Statistical analysis}

Data were analysed using $R$ v.3.4.0 ( $R$ Core Team, 2017) and the lme4 package (v. 1.1.23; Bates et al., 2015). Linear mixed-effects models fit by maximum likelihood were used to examine the relationship between individual alpha frequency, emotional valence and sleep on memory consolidation. Mixed models are an appropriate method for analysing data from repeated measure designs, as these designs are grouped by subject and appropriately account for within and between subject variance (Judd et al., 2012; Van Dongen, Olofsen, Dinges \& Maislin, 2004). The behavioural model (i.e., no sleep EEG predictors) took the following structure:

dprime $\sim$ condition $*$ valence $*$ iaf $*$ baseline $+f$ siq $+(1 \mid$ subj $)$

where condition is sleep versus wake, valence is the emotional valence of the stimulus (positive, negative, neutral), iaf refers to individual alpha frequency estimates, baseline is d' at immediate recall, and fsiq refers to a general intelligence quotient estimated from the WASI. Subject ID (subj) was modelled as a random effect on the intercept. The sensitivity index (d'; dprime) from the delayed retrieval task was modelled as the outcome variable. Asterisks denote interaction terms between variables, while + indicates factors entered into the model as fixed effects without interaction terms.

Critically, the inclusion of baseline performance in the model controls for baseline memory performance at the immediate testing session. This approach also reduces the amount of variance in the residual error term, increases statistical power, and has been adopted as an alternative baselining approach, as for example, in event-related potential research (Alday, 2019; see also Cross et al., 2020). Further, the FSIQ scores (estimated from the WASI-II) were added into the model as a fixed effect, given that individual differences in intelligence modulate a range of cognitive processes, including sleep-related memory consolidation (Fenn and Hambrick, 2015), and is correlated with IAF (e.g., Grandy et al., 2013a).

Similarly, the sleep EEG model took the following structure:

dprime_dif $\sim$ iaf $*$ rem $* s w s *$ valence $+f s i q+(1 \mid s u b j)$

where iaf refers to individual alpha frequency estimates, rem is REM theta power, sws is SWS SO density, valence is the emotional valence of the stimulus (positive, negative, neutral), and $f$ siq refers to the general intelligence quotient estimated from the WASI. The random effects consisted of intercept by subject. The difference in d' scores between the immediate (B) and delayed (D) retrieval tasks was modelled as the outcome variable $(D-I=$ dprime_dif $)$. Difference scores in d' were used in the sleep model as the model failed to converge with d' scores from delayed testing as the outcome and baseline d' as a fixed effect, likely based on the smaller number of observations compared to the behavioural model given that the observations nested under the wake condition were removed. All categorical variables used sum-to-zero coding, with the reference level set to -1 . Please also note that more complex random effect structures involving random slopes by participant did not converge.

Akaike Information Criterion (AIC; Akaike, 1974) was used to assess model fit, while Type II Wald Chi-Square $(\chi 2)$ tests from the car package (Fox and Weisberg, 2011) were used to provide $p$-value estimates for each of the factors. All $p$-values are 2-tailed, with statistical significance determined at $\alpha=0.05$. All data are presented as mean and standard error (SEM) unless indicated otherwise, and effects were plotted using the package ggplot2 (Wickham, 2009). Raincloud plots were produced to visualise behavioural data using the code provided by Allen et al. (2019). In order to isolate outliers, we used Tukey's method, which identifies outliers as exceeding $\pm 1.5 *$ inter-quartile range. Graphical displays of modelled effects include $83 \%$ confidence intervals (CI), the non-overlap of which corresponds to the $95 \%$ significance level of the difference (Austin and Hux, 2002; MacGregor-Fors and Payton, 2013). Visualisations of the raw data (e.g., scatterplots) used the conventional $95 \%$ confidence interval, given that these did not include contrasts between multiple moderating variables and associated significance testing of group differences. Finally, we used a "small multiples" approach to visualise complex interactions from the linear mixed-effects models. Small multiples enable clearer visualisation of complex interaction effects by slicing data into multiple related grids, thus avoiding over-plotting and facilitating exploration of the whole dataset (van den Elzen and Wijk, 2013; Tufte, 1983).

\section{Results}

\subsection{Preliminary analyses}

Preliminary analyses were conducted to determine whether there were differences in levels of self-perceived sleepiness between the Sleep and Wake conditions prior to the Immediate and Delayed recall tasks, and to report the sleep characteristics of the nap and the distribution of IAF estimates.

Subjects were significantly sleepier during the Sleep $(M=54.57, S D$ $=18.53)$ than the Wake $(M=64.43, S D=21.23)$ condition prior to the learning and immediate recall tasks $(t(20)=2.26, p<.001, d=-0.46)$. There was no difference between the conditions at delayed recall (Sleep: $M=61.47, S D=16.42$, Wake: $M=67.64, S D=15, t(20=-1.23, p>$ $.05, d=-0.39$ ). A linear regression was conducted to determine whether the greater levels of sleepiness in the Sleep group impacted immediate memory performance. The results of the regression indicate that there was no significant effect of self-reported sleepiness on immediate memory performance $\left(\beta=-0.71, p=.66, R^{2}=-0.04\right)$.

IAF estimates varied among participants, with a mean IAF estimate of $10.40 \mathrm{~Hz}$ and a range of $8.50-12.35 \mathrm{~Hz}$. Peak alpha frequency estimates from both the Sleep and Wake conditions are displayed in Fig. 4.

Sleep variables of total sleep time, sleep onset latency, wake after sleep onset and the amount of time and percentage of sleep spent in stage 1 (N1), stage 2 (N2), SWS and REM are reported in Table 2. Sleep data show the expected proportion of NREM sleep stages (i.e., N1, N2 and SWS) and minimal REM sleep (Payne et al., 2015). That is, although 81 percent of participants experienced REM, average time spent in this stage was only approximately $8 \mathrm{~min}$.

\subsection{Primary analysis}

4.2.1. Memory consolidation across sleep and wake is modulated by IAF

The results of the immediate and delayed recall tests are given in Table 3 and Fig. 4. Nine of 244 d' observations were classed as outliers 

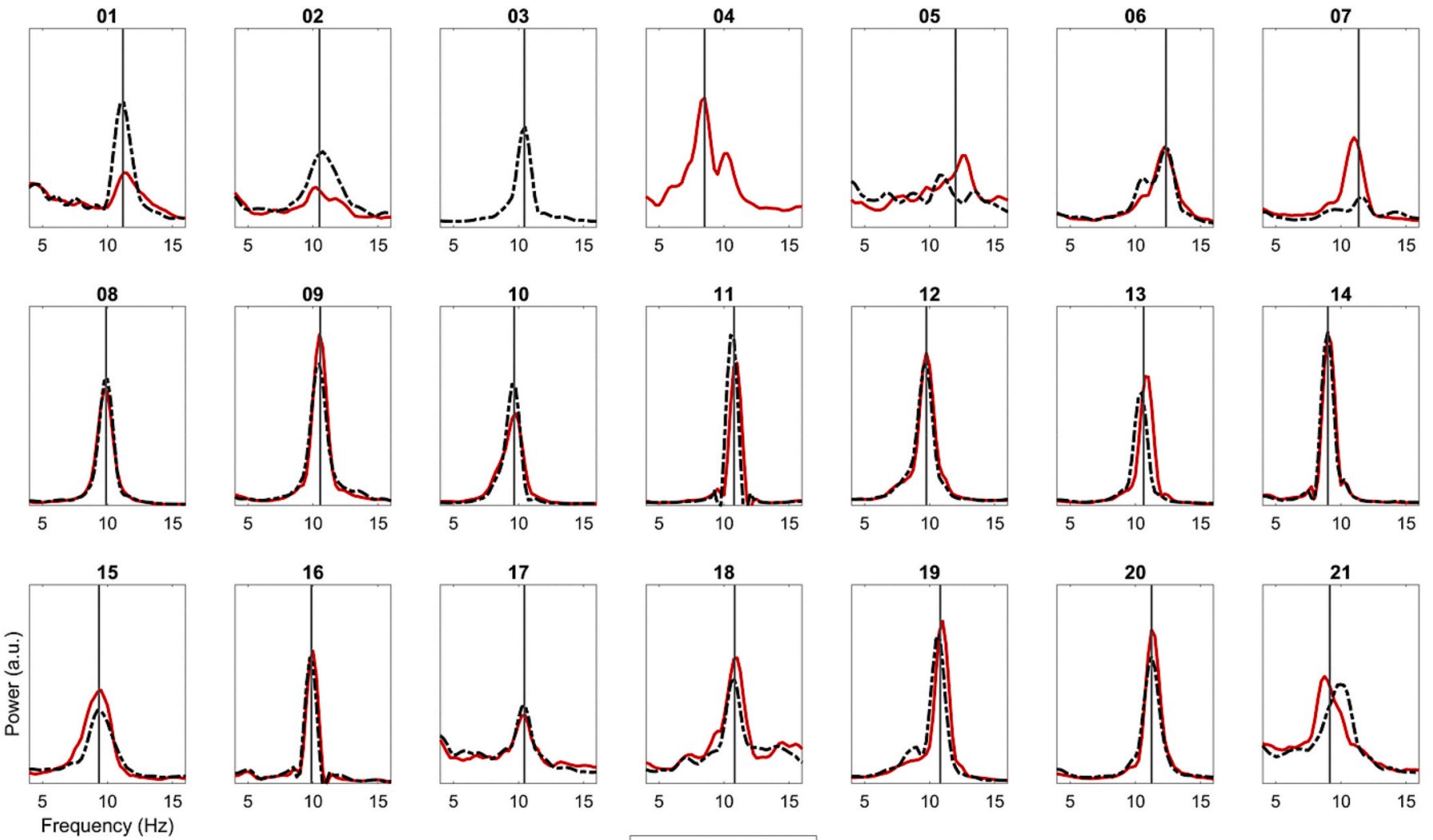

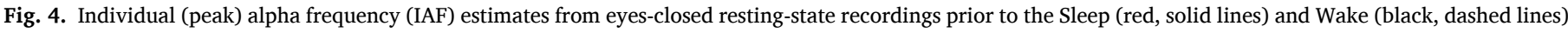

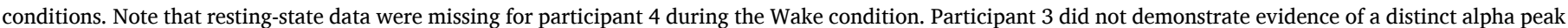

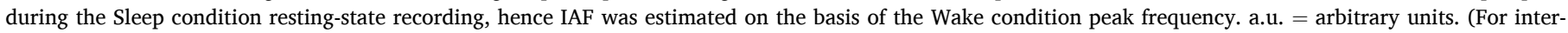
pretation of the references to colour in this figure legend, the reader is referred to the Web version of this article.)

Table 2

Descriptive statistics for sleep parameters and correlations with the difference between d' at delayed and baseline testing as a proxy for memory change.

\begin{tabular}{|c|c|c|c|c|c|}
\hline \multirow{2}{*}{$\begin{array}{l}\text { Sleep } \\
\text { Parameters }\end{array}$} & \multirow{2}{*}{$\begin{array}{l}\text { Mean } \\
\text { Minutes } \\
\text { (SEM) }\end{array}$} & \multirow{2}{*}{$\begin{array}{l}\% \text { in Stage } \\
(\mathrm{SEM})\end{array}$} & \multicolumn{3}{|c|}{ Correlations with d' } \\
\hline & & & $\mathrm{r}$ & $\begin{array}{l}p \\
\text { (uncorrected) }\end{array}$ & $\begin{array}{l}p \\
\text { (Holm) }\end{array}$ \\
\hline TST & $91.45(5.76)$ & & .13 & .31 & 1.00 \\
\hline SOL & 13.64 (3.07) & & -.20 & .12 & .77 \\
\hline WASO & $15.10(3.86)$ & & -.06 & .64 & 1.00 \\
\hline N1 & $7.07(1.20)$ & $8.12(1.19)$ & -.44 & $<.001$ & .005 \\
\hline N2 & $46.76(3.67)$ & $\begin{array}{l}51.78 \\
(2.92)\end{array}$ & -.26 & .04 & .32 \\
\hline sWs & $29.04(3.53)$ & $\begin{array}{l}31.33 \\
(3.52)\end{array}$ & .21 & .11 & .77 \\
\hline REM & $8.57(1.73)$ & $8.77(1.72)$ & .30 & .02 & .18 \\
\hline $\begin{array}{l}\text { EEG } \\
\text { Parameter }\end{array}$ & $\begin{array}{l}\text { Mean } \\
\text { (SEM) }\end{array}$ & Range & & & \\
\hline $\begin{array}{l}\text { SWS Spindle } \\
\text { Density }\end{array}$ & $2.18(0.12)$ & $1.11-3.10$ & -.07 & .61 & 1.00 \\
\hline $\begin{array}{l}\text { SWS SO } \\
\text { Density }\end{array}$ & $2.27(0.10)$ & $1.44-3.17$ & -.08 & .55 & 1.00 \\
\hline $\begin{array}{l}\text { REM Theta } \\
\text { Power }\end{array}$ & $4.11(0.40)$ & $1.76-7.43$ & .11 & .44 & 1.00 \\
\hline
\end{tabular}

Note. SEM = standard error of the mean. TST $=$ total sleep time; SOL = sleep onset latency; WASO = wake after sleep onset; $\mathrm{N} 1=$ stage 1 ; N2 = stage 2; SWS = slow wave sleep; REM = rapid eye movement sleep; SWS = slow wave sleep; $\mathrm{REM}=$ rapid eye movement sleep. Correlations represent the relationship between percent of time spent in each sleep stage and d'. Significance values in the last column on the right are Holm-Bonferroni corrected (Holm, 1979).
Table 3

Mean d' scores by Condition (sleep, wake), Time (immediate, delayed) and Valence (positive, negative, neutral). Standard errors are given in parentheses.

\begin{tabular}{llll}
\hline Condition & Time & Valence & Mean (SEM) \\
\hline Sleep & Immediate & Negative & $2.23(.10)$ \\
& & Neutral & $2.64(.12)$ \\
& & Positive & $2.43(.14)$ \\
& Delayed & Negative & $1.79(.13)$ \\
& & Neutral & $2.19(.15)$ \\
Wake & Positive & $2.08(.16)$ \\
& Immediate & Negative & $2.35(.14)$ \\
& & Neutral & $2.55(.15)$ \\
& Delayed & Pesitive & $2.55(.15)$ \\
& & Negative & $1.76(.17)$ \\
& & Neutral & $1.91(.21)$ \\
& & Positive & $1.84(.17)$ \\
\hline
\end{tabular}

Note. SEM $=$ standard error of the mean.

and were removed prior to calculating d' difference scores (the distribution of data before and after the removal of outliers is depicted in Fig. S1). The sleep condition had a higher overall d' score at delayed testing compared to the wake condition. Fig. 5A also demonstrates that the sleep condition had less broadly distributed d' scores across the valence categories, particularly for negative stimuli, relative to the wake condition.

In order to determine whether emotional memory consolidation differed over a period of sleep and wake, we examined whether Valence (positive, negative, neutral) and Condition (sleep vs wake) modulated d' scores. Linear mixed-effects modelling revealed a main effect of Condition $(\chi 2(1)=8.04, p=.004)$, with the Sleep condition having a higher d' score relative to the Wake condition. This effect is illustrated in Fig. 5B, 
(A)

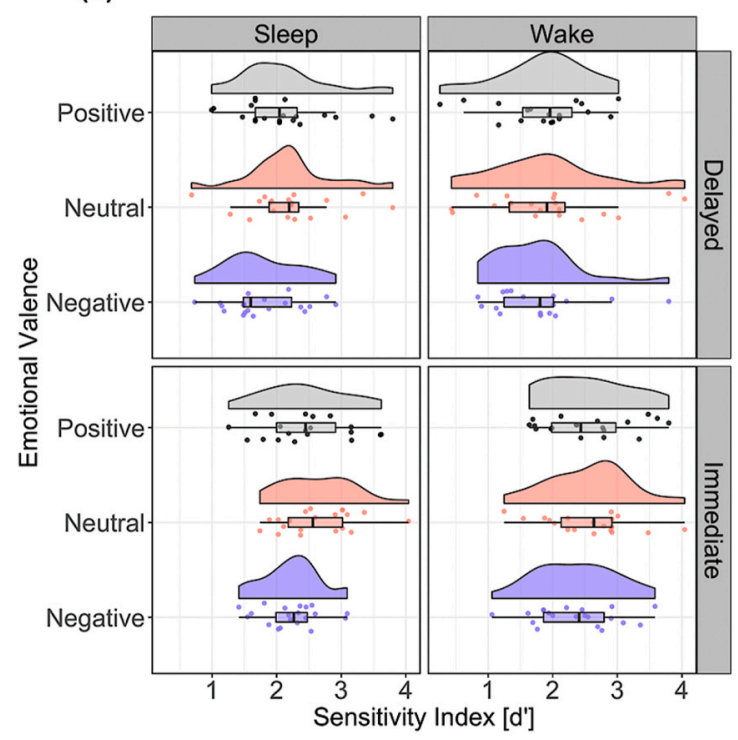

(B) Valence $\rightarrow$ Negative $\rightarrow$ Neutral $\rightarrow$ Positive

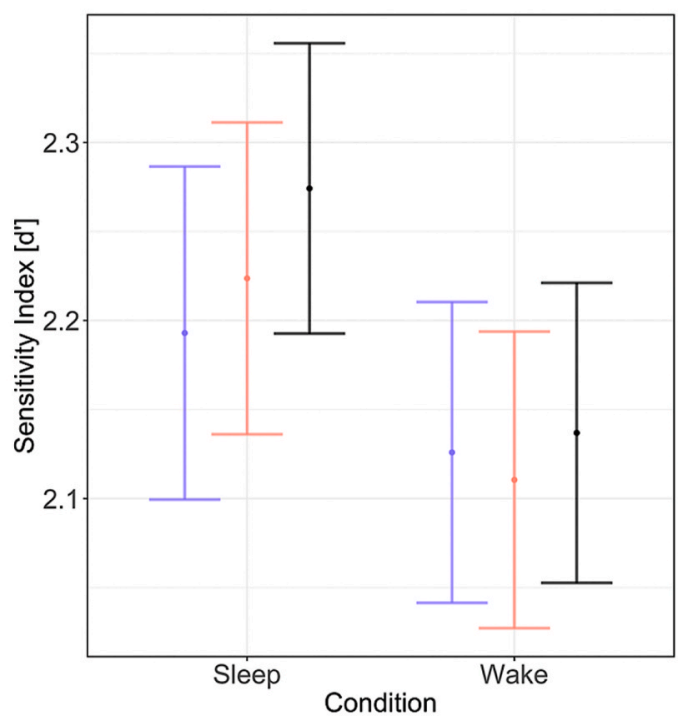

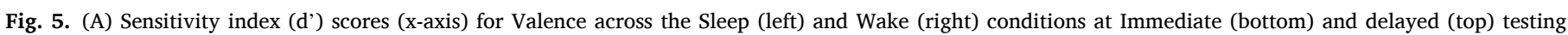

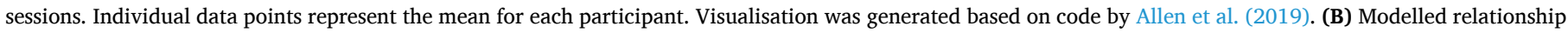
between d' scores at delayed testing, Condition (sleep, wake; x-axis) and Valence (negative, neutral, positive).

where the sleep condition had higher d' scores for all three valence categories compared to the wake group, suggesting sleep preserves memory to a stronger degree relative to an equivalent period of wake, and supporting $\mathrm{H}^{1}$ that memory performance (d') would be greater after sleep compared to wake.

The main effect of Valence $(\chi 2(2)=1.68, p=.43)$ and the Condition $\times$ Valence interaction $(\chi 2(2)=0.78, p=.67)$ were nonsignificant, suggesting that any potential effect of emotional valence is sufficiently small or variable that we could not detect its impact on behavioural memory performance across the 2-hr afternoon nap compared to an equivalent period of wake (Lehmann et al., 2016). Thus, $\mathrm{H}^{1}$ that memory would be greater after sleep compared to wake was supported; however, $\mathrm{H}^{2}$ that memory would be greater for emotional compared to neutral information and this effect would be accentuated after sleep compared to wake was not supported.

Next, we examined whether IAF explains differences in memory between the sleep and wake conditions. Critically, there was a significant Condition $\mathrm{x}$ Time $\mathrm{x}$ IAF $\times$ Baseline interaction $(\chi 2(1)=6.15, p=$ .01 ), which is resolved in Fig. 6. In both sleep and wake conditions, higher baseline d' scores predicted higher delayed d' scores. For the wake condition, higher IAF estimates were associated with lower d' scores (i.e., worse memory) irrespective of baseline memory performance. However, for the sleep condition, a higher IAF estimate predicted improved d' scores at delayed testing when baseline memory performance was low, while the reverse was observed when baseline memory performance was high.

Together, these results indicate that IAF differentially modulates memory across a period of sleep relative to wake, such that a higher IAF may facilitate sleep-related memory consolidation when baseline memory performance is suboptimal. By contrast, when baseline memory performance is high, we see a beneficial influence of low IAF on sleeprelated memory consolidation (for a full summary of all main effects, interactions and a model summary, see Tables S1 and S2 in the supplementary materials, respectively).

\subsubsection{Individual alpha frequency modulates spindle-related emotional memory consolidation}

To examine whether the positive behavioural effect of sleep on memory was driven by underlying neural oscillatory mechanisms, we examined the effect of IAF, sleep spindle density and SO density, and
REM theta power on d' scores for each valence category. The first model focussed on the interaction between IAF, SWS Spindle Density and Valence. Importantly, the IAF $\mathrm{x}$ Spindle Density $\times$ Valence interaction was significant $(\chi 2(2)=6.64, p=.03)$, which is resolved in Fig. 7. As is clear from Fig. 7, when IAF is low (i.e., $9.5 \mathrm{~Hz}$ ) and Spindle Density is high, memory retention for negative and neutral stimuli is low. This pattern of results reversed when IAF was high (i.e., $11 \mathrm{~Hz}$ ): an increase in IAF and Spindle Density predicted an increase in memory scores for negative and neutral stimuli, but predicted a decrease in memory for positive stimuli. Together, these results suggest that IAF modulates spindle-related memory consolidation of stimuli differing in emotional valence, partially addressing our research question of whether IAF and sleep physiology interact to influence memory (for a full summary of all main effects, interactions and a model summary, see Tables S3 and S4 in the supplementary materials, respectively).

\subsubsection{Slow oscillations, REM theta power and individual alpha frequency jointly predict memory}

Given the proposed individual and interactive roles of slow wave and REM sleep in the consolidation of (emotional) memory, and the influence of IAF on information processing, we now examine whether: (1) IAF modulates the separate effects of slow wave and REM sleep on emotional memory consolidation, and; (2) whether the interactive effect of slow wave and REM sleep on emotional memory consolidation is modulated by IAF. The broad purpose of this analysis was to examine our research question of whether IAF and sleep physiology interact to influence memory.

We implemented a linear mixed-effects model to examine changes in memory across sleep, quantified as changes in d' from immediate to delayed testing, as a function of Valence (negative, neutral, positive), IAF, SWS Slow Oscillation Density and REM Theta Power. The random effects consisted of intercept by subject. There was a main effect of IAF $(\chi 2(1)=28.24, p<.001)$, with a higher IAF predicting a negative change in d' scores. There were also significant main effects of SWS Slow Oscillation Density $(\chi 2(1)=8.41, p=.003)$ and REM Theta Power $(\chi 2$ $(1)=38.64, p<.001$ ), with higher SO density predicting negative changes in d' scores, while greater REM theta power was associated with a positive change in d' scores (for visualisation of main effects, see Fig. 8).

We also observed a significant IAF x SWS Slow Oscillation Density 


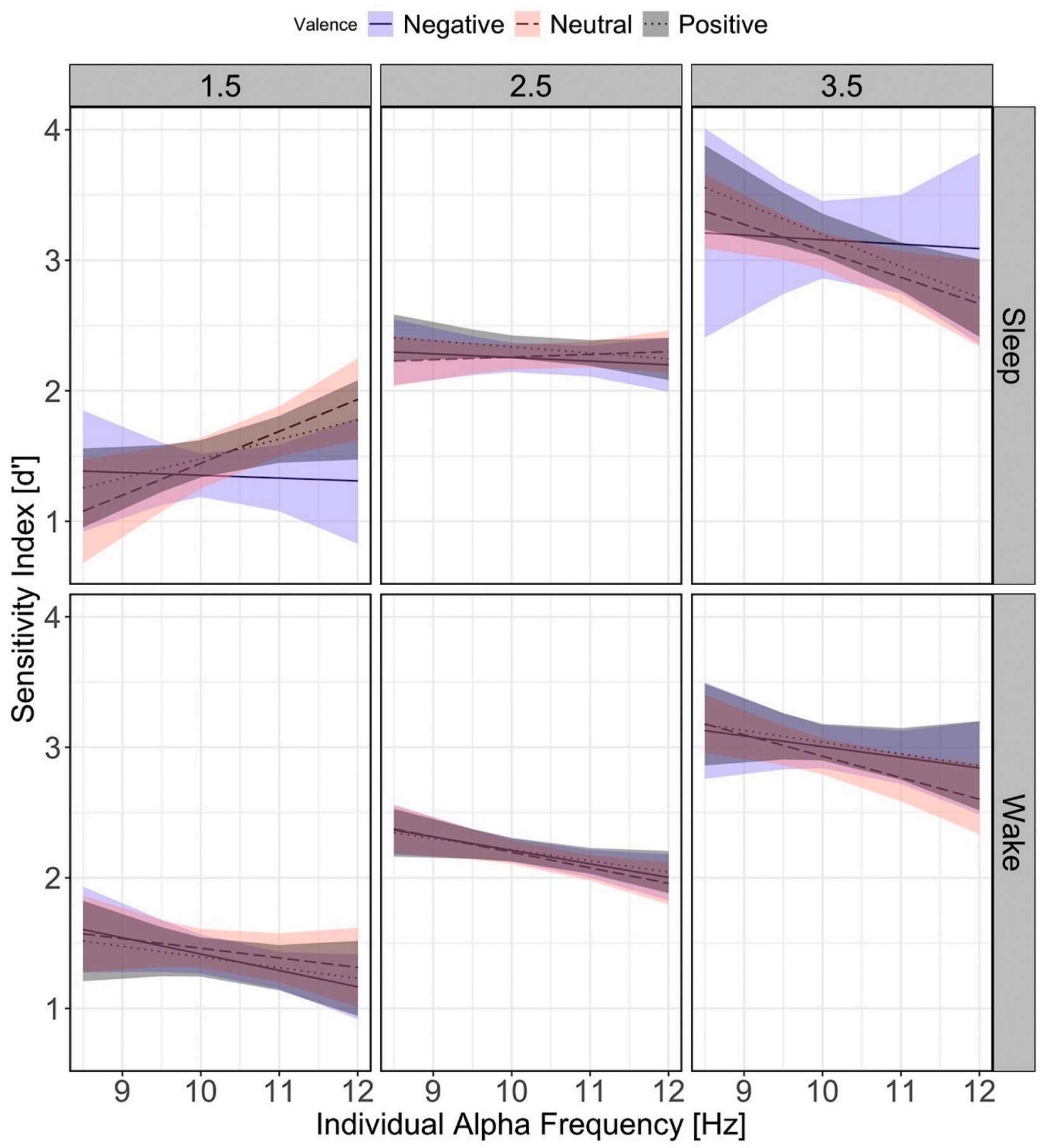

Fig. 6. | Estimated marginal means for d' scores at delayed testing by IAF ( $\mathrm{x}$-axis) and valence (negative $=$ purple solid line, neutral = dashed pink line, positive $=$ grey dotted line). Facets represent predicted values per unit increase in baseline d' scores from low (left, 1.5) to high (right, 3.5). Shaded regions indicate the 83\% CI. (For interpretation of the references to colour in this figure legend, the reader is referred to the Web version of this article.)

interaction $(\chi 2(1)=5.73, p=.01)$. As shown in Fig. 9A, low IAF values and high SO density predicted lower d' scores, while this effect reduced with high IAF and high SO density estimates. Further, REM Theta Power, SWS Slow Oscillation Power and IAF interacted to predict d' scores $(\chi 2$ $(1)=5.71, p=.01)$. As shown in Fig. 9B, low SWS SO density, low IAF and high REM theta power predicted high d' scores, while high IAF, high SWS SO density and REM theta power had little effect on d' scores. These results suggest that IAF differentially modulates the separable effect that SWS and REM have on the consolidation of emotional information, and that SWS and REM interact to predict memory consolidation.

Critically, the four-way interaction between IAF, REM Theta Power,
SWS Slow Oscillation Power and Valence was also significant $(\chi 2(2)=$ $10.39, p=.005)$. As shown in Fig. 10 , IAF was positively related to memory retention for positively valenced stimuli when SWS SO density was high and REM theta power was low (bottom left panel). This relationship reverses when REM theta power and IAF are high and SWS SO density is low (top right panel; for a full summary of all main effects, interactions and a model summary, see Tables S5 and S6 in the supplementary materials, respectively). 


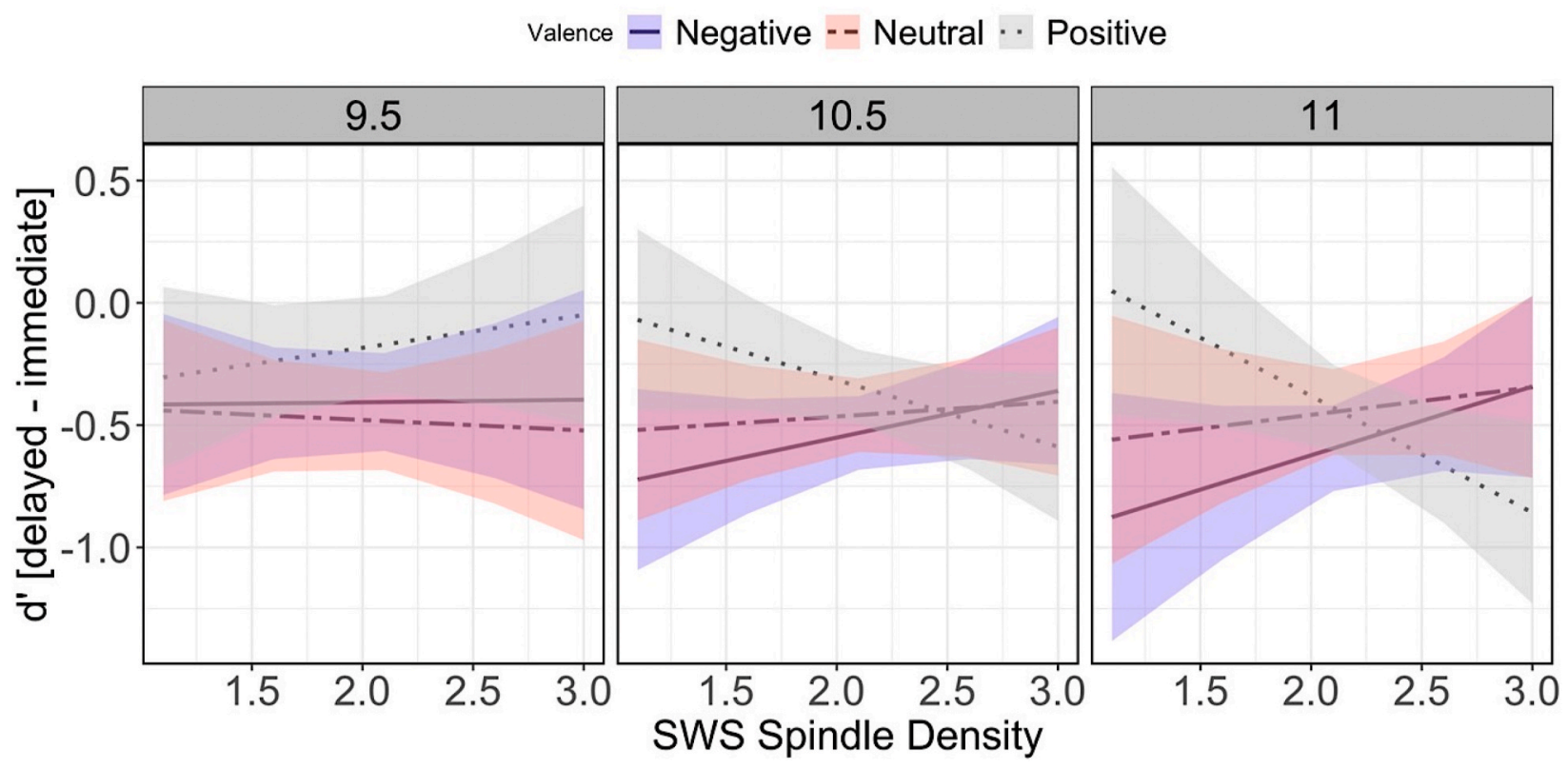

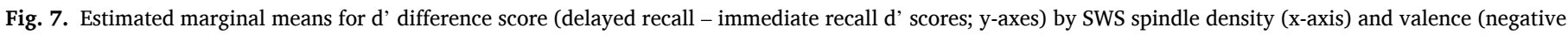

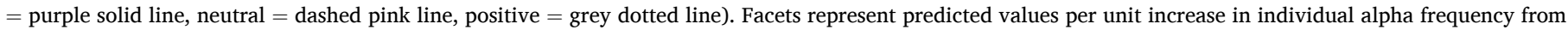

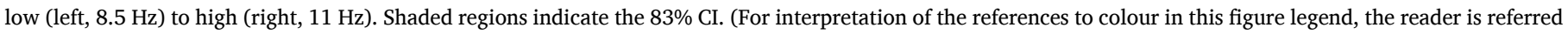
to the Web version of this article.)

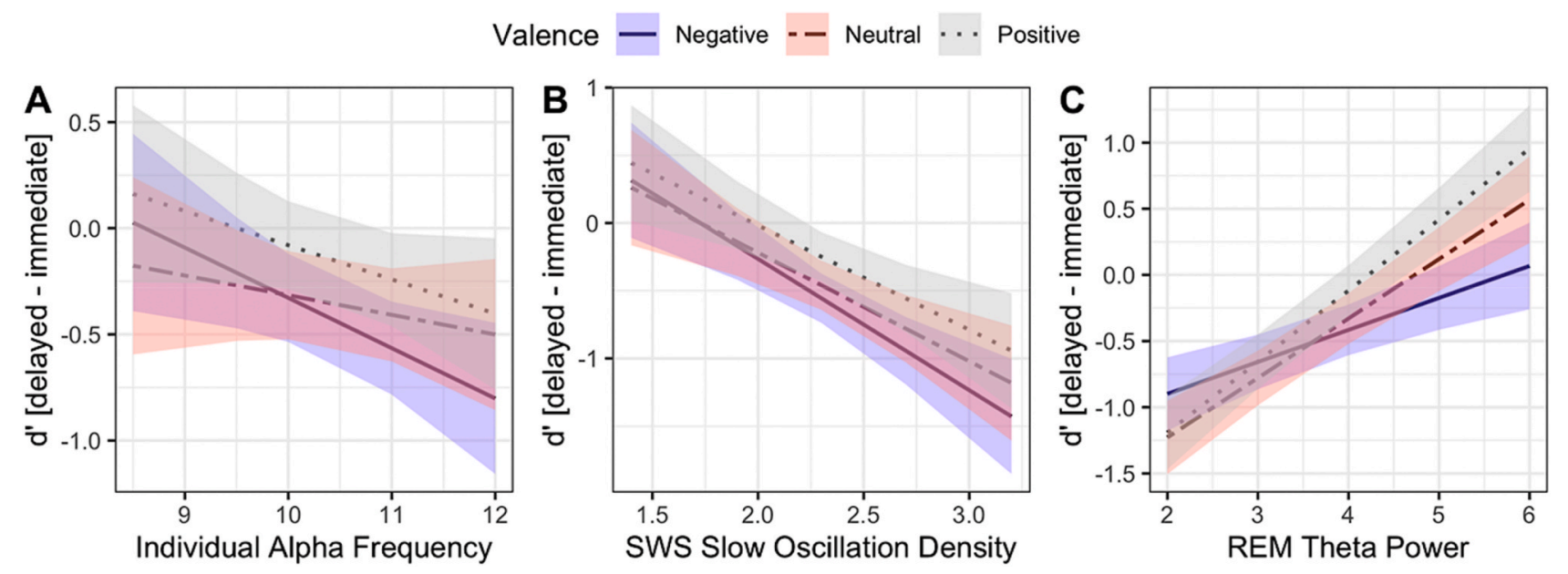

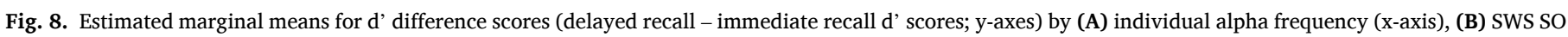

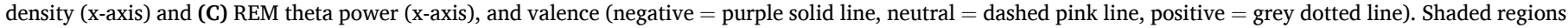
indicate the $83 \%$ CI. (For interpretation of the references to colour in this figure legend, the reader is referred to the Web version of this article.)

\subsubsection{Relationship between individual alpha frequency and sleep parameters}

As an exploratory analysis, we examined correlations between IAF estimates and sleep EEG metrics to assess whether individual differences in IAF predicts sleep oscillatory activity. We did not correct for multiple comparisons, as to not imply that our analyses were confirmatory. Instead, we focus on effect size estimates, and report all relevant statistical information. This supports recommendations for exploratory analyses to focus on effect size estimates (e.g., $r, R^{2}$ ), rather than on significance levels (Szucs and Ioannidis, 2017; also see Jach et al., 2020 for a similar approach). There was a small positive correlation between spindle density and IAF $(r=0.19, p=.42,95 \% \mathrm{CI}=[-0.28,0.60])$, and a moderate positive correlation between spindle amplitude and IAF $(r=$ $0.43, p=.06,95 \% \mathrm{CI}=[-0.02,0.74])$. There was also a moderate positive correlation between SWS SO density and IAF ( $r=0.31, p=.19$, $95 \% \mathrm{CI}=[-0.16,0.67])$; however, there was a large positive correlation between REM theta power and IAF $(r=0.64, p=.005,95 \% \mathrm{CI}=[0.23$, 0.85]). These relationships are illustrated in Fig. 11.

\section{Discussion}

Here, we paired an affective memory task with EEG and a nap paradigm in order to determine the relationships between the emotional valence of encoded stimuli, individual EEG factors related to information processing and sleep in predicting memory outcomes. The results of this study inform these relationships, indicating a significantly greater preservation of encoded material across sleep relative to a wake period, regardless of the emotional valence of the material. Results also inform our understanding of the role of individual EEG factors in sleep and memory consolidation, indicating a role of IAF in modulating the effects of sleep micro- and macro-structural variables in determining memory outcomes. Collectively, these results highlight the importance of 

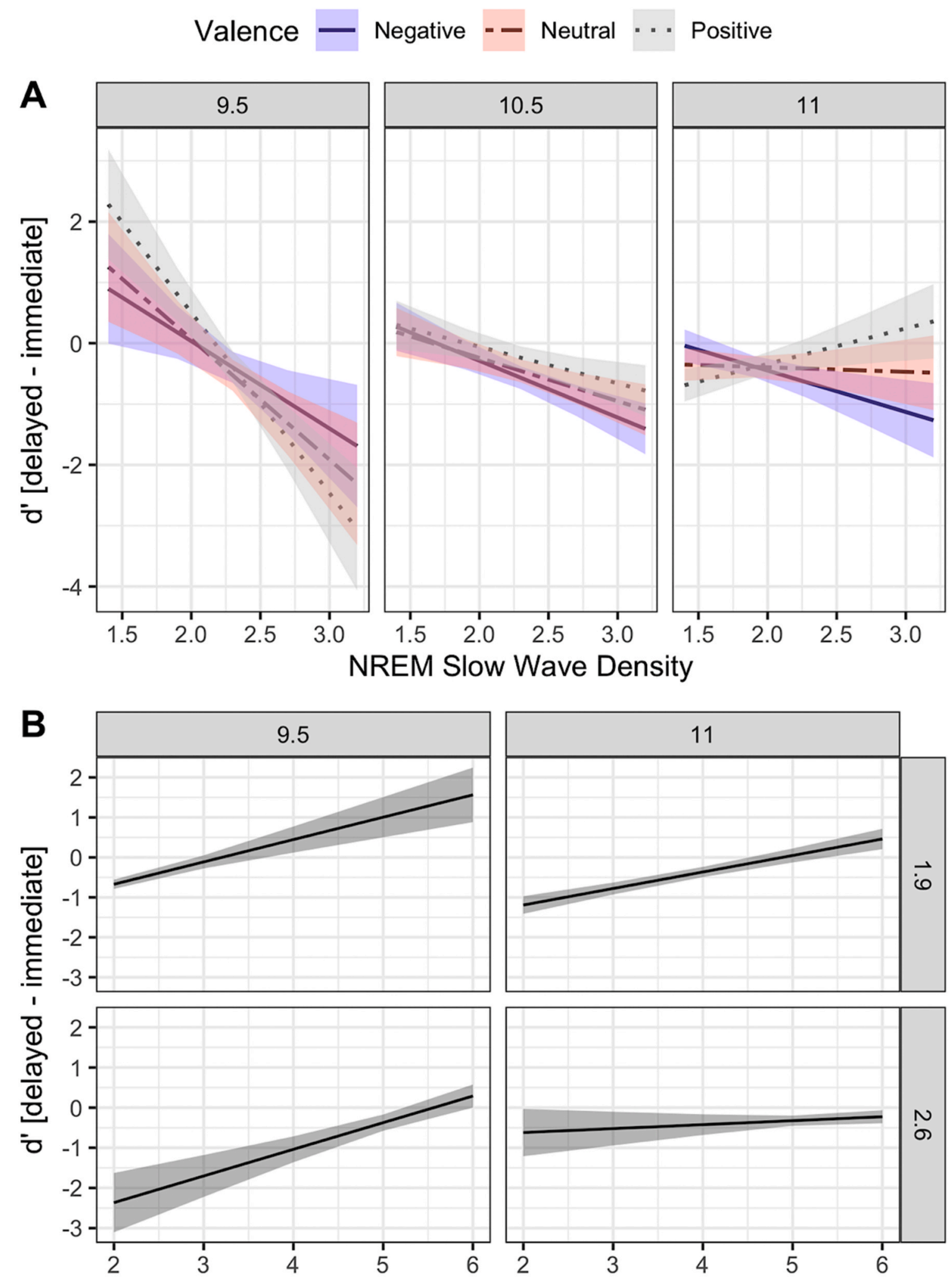

REM Theta Power

Fig. 9. (A) Estimated marginal means for d' difference score (delayed recall - immediate recall d' scores; $y$-axes) by SWS SO density ( $x$-axis) and valence (negative = purple solid line, neutral = dashed pink line, positive = grey dotted line). Facets represent predicted values per unit increase in individual alpha frequency from low (left, $9.5 \mathrm{~Hz}$ ) to high (right, $11 \mathrm{~Hz}$ ). (B) Estimated marginal means for d' difference score (delayed recall - immediate recall d' scores; y-axes) by REM theta power (xaxis). Facets represent predicted values per unit increase in individual alpha frequency from low (top left, using $9.5 \mathrm{~Hz}$ as a canonical value) to high (top right, using $11 \mathrm{~Hz}$ as a canonical value), and SWS SO density from low (top right, 1.9) to high (bottom right, 2.6). Shaded regions indicate the $83 \%$ CI. 


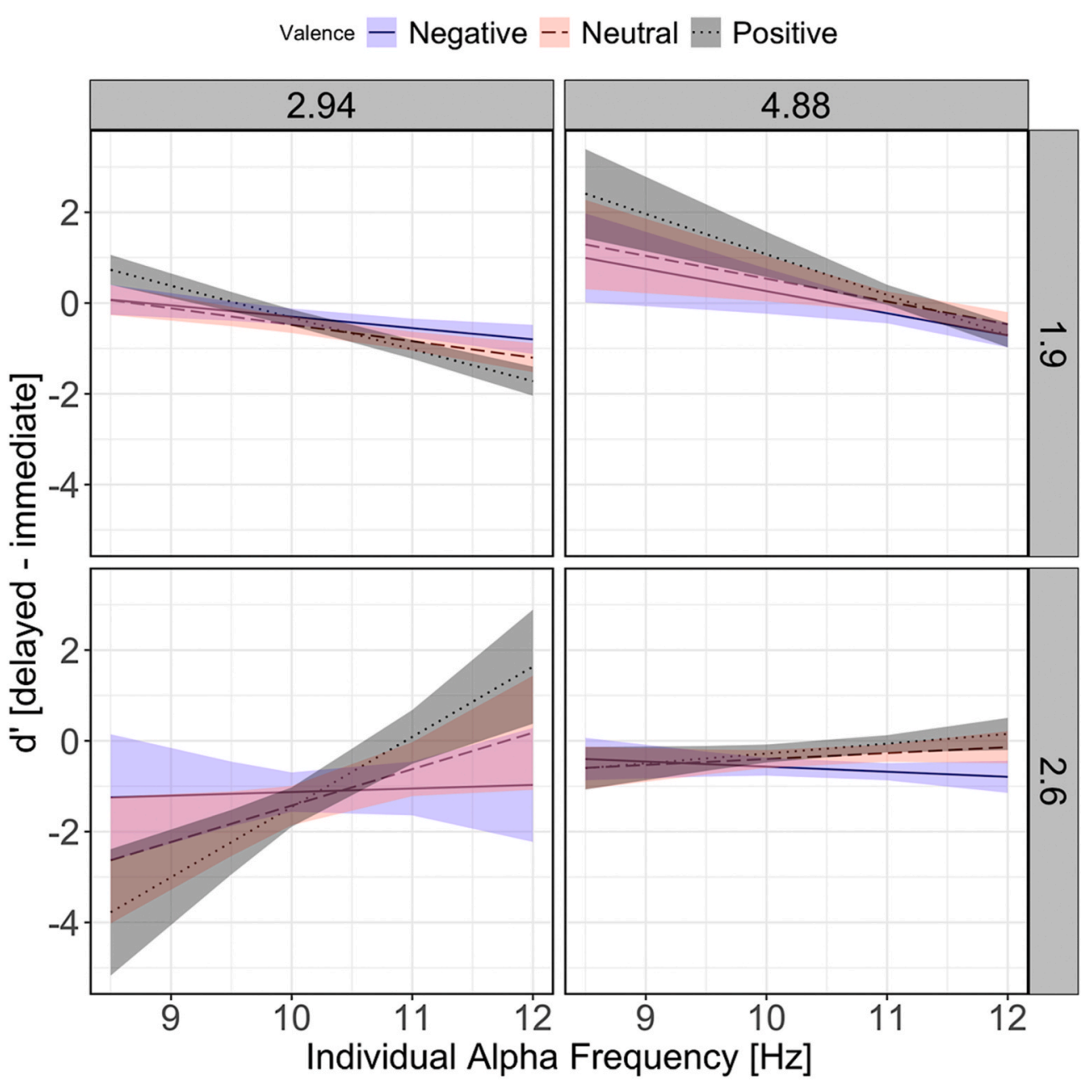

Fig. 10. Estimated marginal means for d' difference score (delayed recall - immediate recall d' scores; y-axes) by individual alpha frequency ( $\mathrm{x}$-axis) and valence (negative = purple solid line, neutral = dashed pink line, positive $=$ grey dotted line). Facets represent predicted values per unit increase in REM theta power (low = top left; high = top right), and SWS SO density from (low = top right; high $=$ bottom right). Shaded regions indicate the $83 \% \mathrm{CI}$. (For interpretation of the references to colour in this figure legend, the reader is referred to the Web version of this article.) individual factors in the EEG in predicting the trajectory a given encoded item will take through sleep-based memory consolidation.

\subsection{The influence of sleep on (emotional) memory consolidation}

Sleep resulted in relatively diminished forgetting, regardless of emotional valence. This is in keeping with previously published work on the broad role of sleep in memory consolidation; the mean differences between our sleep and wake conditions are consistent with the $\sim 10 \%$ improvement in recognition scores, as reported in Rasch and Born (2013). We observed a negative correlation between the percentage of time spent in N1 sleep and d' scores, and this likely reflects N1 sleep as a marker of sleep disruption (Berry et al., 2012), whereby greater N1 likely limited the opportunity for (N)REM sleep-associated memory consolidation. It should be noted, however, that we did not find a significant effect of valence in the consolidation of emotional memories.

There are potentially two main reasons for this. Firstly, participants were aware that they were to be tested on the recognition of encoded images. This expectancy may have interfered with the priority given to images regardless of emotional tone, thus ablating whatever emotional priority may have naturally occurred with the intervention (Groch et al., 2015). Secondly, the negative images used in this study may not have been sufficiently negative to prompt a priority consolidation based on the valence of the images. Similarly, we did not use affective foreground/background imagery (Payne et al., 2012; Payne et al., 2008). Furthermore, the influence of participants' awareness of being tested on learned material and affective valence are known to influence memory performance (Bennion et al., 2016; Groch et al., 2016), and might differentially modulate the effects of sleep and wake. While it has been reported that predictions evoked by intentional coding paradigms do not critically affect sleep groups, they have been found to enhance the rehearsal of target content during waking delay periods (Cunningham et al., 2014). Moreover, cognitive states (e.g., motivation) might have played a more dominant role in organising information processing during sleep (Bennion et al., 2016). Consequently, a sleep relative to a wakeful delay period may have furnished distinct contributions to the consolidation of memorised items. This possibility presents an interesting opportunity for future research. In sum, preferential consolidation of neutral and negative images as found in our higher IAF subjects may be due to processes other than emotional tone.

\subsection{IAF and the modulation of sleep-associated memory consolidation}

Our results indicate a complex relationship between IAF, REM theta power, SO density and sleep spindle density in determining the success or failure of recognition of encoded images across sleep. In both behavioural and sleep-oscillation based models, high IAF individuals recognised more neutral and positive images than low IAF individuals. This is the first study to report categorical differences in the operation of memory consolidation based on individual differences in the EEG, and there are two main potential explanations of this observation: first, that higher IAF individuals have faster (higher average frequency) brain activity in general, thus allowing more iterations of trace replay in the same amount of time; and thereby greater memory retention. We have also noted that IAF does not modulate differences in the recollection of negative imagery. It could be proposed that negatively valenced 

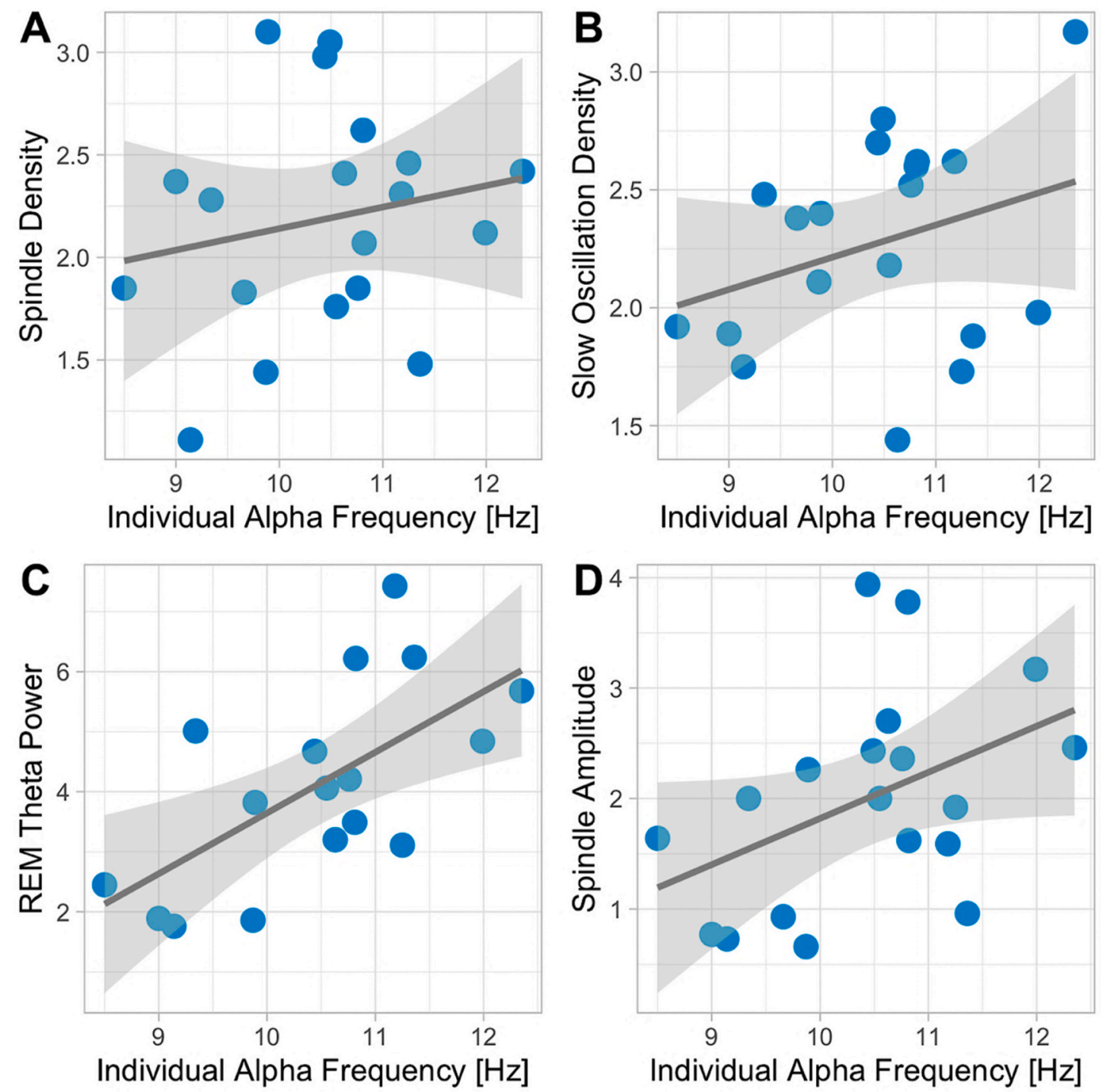

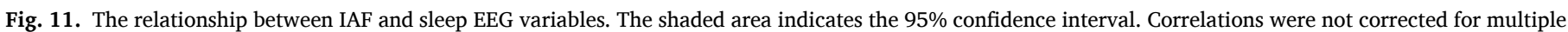
comparisons given the exploratory nature of the analysis and small number of comparisons.

memories are the more efficacious items for the human brain to encode, and that the greater recognition of neutral and positive memories demonstrated by high IAF individuals results from faster trace replay allowing more room for 'less important' memories to be consolidated in comparison to lower IAF individuals.

The prominent spectral peak of IAF has been suggested for use as a landmark for the custom-fitting of individual frequency band cut-offs in the EEG (Klimesch, 1999). A higher IAF individual will therefore have higher boundaries of EEG frequency bands in comparison to a lower-IAF individual. While the functional implications of this have not been studied, it is possible that small improvements in the fit of oscillatory dynamics (such as the coupling of hippocampal sharp-wave ripples and sleep spindle troughs; Staresina et al., 2015) may result in small benefits in the behavioural outcomes associated with them. The functional consequences of IAF categories in terms of neural dynamics are an area which should be studied in the future; a basic investigation of spindle mean frequency as a function of IAF would be an excellent start thereon.

A second explanation for this interaction is that higher IAF values may allow the brain to preferentially encode salient information. This would explain the behavioural findings in line with work on emotional memory (e.g., Vuilleumier, 2005), but this leads to several greater open questions in the sleep and memory literature, namely how a given item is tagged for subsequent consolidation. Current work in this area has suggested that emotional salience (Payne et al., 2008), as well as schema conformance (Durrant et al., 2015) may tag items for consolidation, and immediate recall theta power may serve as a biomarker of this tagging (Heib et al., 2015), through an interaction with fast sleep spindle activity. However, in general, there is a poor understanding of the encoding-related EEG factors which predispose an item to be remembered, forgotten or generalized. A promising next step to address this issue would be to pair sleep and memory paradigms with a subsequent memory approach in order to measure the differential patterns of EEG between remembered and forgotten items (as well as those gained overnight).

\subsection{Individual alpha frequency, REM theta and slow oscillatory activity}

We have also noted a relationship between REM theta power and IAF, which modulated the recognition of emotional images. Although we observed a linear increase in REM theta power as a function of IAF, the functional effect of this in terms of recognition appears to be centred around lower-IAF subjects. There is relatively less data published in the 
literature on the role of REM sleep in memory, although recent accounts (Grosmark and Buzsaki, 2016; Hobson and Friston, 2012) have suggested a role of REM sleep in synaptic downscaling (although note also Tononi and Cirelli, 2014 for a SWS-based account of the same). This is an attractive explanation for our findings regarding our lower-IAF subjects: it is possible that lower IAF subjects required synaptic downscaling to improve signal to noise ratios and thus obtain a behaviourally measurable effect on (emotional) memory recognition; however, this will need to be explicitly tested in future work.

It should also be noted that our data indicate a potentially complex relationship between REM theta power, SO density and IAF in determining memory outcomes. SOs are argued to be involved in consolidating encoded memory representations (Rasch and Born, 2013). High IAF individuals may therefore be more efficient at encoding discrete stimulus details, allowing SWS memory processes to consolidate a greater amount of information. In terms of explaining the four-way interaction, it is possible that a higher IAF may result in higher resolution sampling of stimulus details during encoding, and higher SO density resulted in relatively strengthened memory traces across sleep. Low REM theta may have indicated a lack of generalisation of encoded traces (potentially due to an inhibition of spreading activation thought to arise from desynchronization of cortex from the hippocampus; Lewis et al., 2018), leading to a preferential remembrance of veridically encoded memories (for similar findings of REM theta on emotional memory, see Kim et al., 2020).

\subsection{Limitations and future directions}

As discussed, one limitation of the current study was minimal REM sleep occurring during daytime naps, as well as large variability in the time participants slept. These effects make it difficult to establish a true effect of REM sleep. Future research using a nocturnal half-night paradigm would involve presenting participants emotional stimuli before either a SWS or REM-rich sleep interval (Groch et al., 2013). Nocturnal half-night paradigms account for natural human nocturnal sleep architecture, and would allow for the systematic investigation between IAF and memory consolidation during SWS and REM.

The adoption of a nocturnal half-night paradigm would be complemented by the use of individual valence reports instead of normalised ratings. While the majority of research utilising the IAPS has relied on normalised ratings (Bradley and Lang, 1994; Lang and Bradley, 2007), there may be large inter-individual variability in the way subjects perceive stimuli along the dimensions of valence and arousal (Backs et al., 2005). Quantifying emotional memory based on individual self-report ratings may increase model sensitivity and the probability of detecting emotion-enhanced memory effects, similar to previous emotional memory research (for review: Talmi, 2013).

\subsection{Conclusions}

Here, we have used an emotional memory task and EEG to highlight several mechanics of memory consolidation in the sleeping brain. We have indicated a role of IAF in modulating memory consolidation, in coordination with REM theta power and SO density in SWS. Individual differences in EEG parameters are rarely considered in sleep and memory research, and from this perspective, IAF may provide a valuable pathway for investigating inter-individual differences in neural correlates of sleep-facilitated memory consolidation. We have also been able to comment on how individual differences in the EEG may influence other elements of sleep neurophysiology, and this has also seldom been studied in the literature. Future research should be sure to account for individual differences as an important factor in sleep and memory research.

\section{Author contribution statement}

ZC - Conceptualization, Methodology, Investigation, Neurophysiological data processing, Statistical Analysis, Writing - Original Draft, Writing - review \& editing, Visualisation. AS - Conceptualization, Methodology, Writing - review \& editing. AWC - Neurophysiological data processing, Writing - review \& editing. AC - Writing - Original draft, Writing - review \& editing. PA - Statistical analysis, Writing review \& editing. SC - neurophysiological data processing, Writing review \& editing. MJK - Conceptualization, Methodology, Writing review \& editing, Supervision.

\section{Declaration of competing interest}

The authors declare no competing financial interests.

\section{Acknowledgements}

We thank Professor Ina Bornkessel-Schlesewsky for helpful comments on an earlier version of this manuscript. We also thank our participants for their enthusiastic participation. AWC is supported by an Australian Government Research Training Program (RTP) scholarship.

\section{Appendix A. Supplementary data}

Supplementary data to this article can be found online at https://doi. org/10.1016/j.neuropsychologia.2020.107660. All data, signal processing and statistical analysis scripts are available at https://osf.io/4 m5hr/?view_only=050d6dd270a24490aa683d883a11f937.

\section{References}

Akaike, H., 1974. A new look at the statistical model identification. IEEE Trans. Automat. Contr. 19 (6), 716-723. https://doi.org/10.1109/TAC.1974.1100705.

Alday, P.M., 2019. How much baseline correction do we need in ERP research? Extended GLM model can replace baseline correction while lifting its limits. Psychophysiology 56 (12), e13451.

Alger, S.E., Lau, H., Fishbein, W., 2012. Slow wave sleep during a daytime nap is necessary for protection from subsequent interference and long-term retention. Neurobiol. Learn. Mem. 98 (2), 188-196.

Allen, M., Poggiali, D., Whitaker, K., Marshall, T.R., Kievit, R.A., 2019. Raincloud plots: a multi-platform tool for robust data visualization. Wellcome open research 4. https:// doi.org/10.12688/wellcomeopenres.15191.1.

Austin, P.C., Hux, J.E., 2002. A brief note on overlapping confidence intervals. J. Vasc. Surg. 36 (1), 194-195.

Backs, R.W., da Silva, S.P., Han, K., 2005. A comparison of younger and older adults' selfassessment manikin ratings of affective pictures. Exp. Aging Res. 31 (4), 421-440.

Bates, D., Maechler, M., Bolker, B., Walker, S., 2015. Fitting linear mixed-effects models using lme4. J. Stat. Software 67 (1), 1-48. https://doi.org/10.18637/jss.v067.i01.

Batterink, L.J., Oudiette, D., Reber, P.J., Paller, K.A., 2014. Sleep facilitates learning a new linguistic rule. Neuropsychologia 65, 169-179.

Bazanova, O.M., Vernon, D., 2014. Interpreting EEG alpha activity. Neurosci. Biobehav. Rev. 44, 94-110.

Bennion, K.A., Payne, J.D., Kensinger, E.A., 2015. Selective effects of sleep on emotional memory: what mechanisms are responsible? Translational Issues in Psychological Science 1 (1), 79.

Bennion, K.A., Payne, J.D., Kensinger, E.A., 2016. The impact of napping on memory for future-relevant stimuli: prioritization among multiple salience cues. Behav. Neurosci. 130 (3), 281.

Berry, R. B., Brooks, R., Gamaldo, C. E., Hardling, S. M., Marcus, C. L., \& Vaughn, B. V. (2012). The AASM Manual for the Scoring of Sleep and Associated Events. Rules, Terminology and Technical Specifications, Darien, Illinois, American Academy of Sleep Medicine.

Born, J., Wilhelm, I., 2012. System consolidation of memory during sleep. Psychological research, Psychological Research 76 (2), 192-203.

Bornkessel-Schlesewsky, I., Philipp, M., Alday, P.M., Kretzschmar, F., Grewe, T., Gumpert, M., et al., 2015. Age-related changes in predictive capacity versus internal model adaptability: electrophysiological evidence that individual differences outweigh effects of age. Front. Aging Neurosci. 7, 217.

Bornkessel, I.D., Fiebach, C.J., Friederici, A.D., Schlesewsky, M., 2004. "Capacity" reconsidered: interindividual differences in language comprehension and individual alpha frequency. Exp. Psychol. 51 (4), 279-289.

Bradley, M.M., Lang, P.J., 1994. Measuring emotion: the self-assessment manikin and the semantic differential. J. Behav. Ther. Exp. Psychiatr. 25 (1), 49-59. https://doi.org/ 10.1016/0005-7916(94)90063-9. 
Buysse, D.J., Reynolds, C.F., Monk, T.H., Berman, S.R., Kupfer, D.J., 1989. The Pittsburgh Sleep Quality Index: a new instrument for psychiatric practice and research. Psychiatr. Res. 28, 193-213.

Cairney, S.A., Durrant, S.J., Hulleman, J., Lewis, P.A., 2014a. Targeted memory reactivation during slow wave sleep facilitates emotional memory consolidation. Sleep 37 (4), 701-707. https://doi.org/10.5665/sleep.3572.

Cairney, S.A., Durrant, S.J., Power, R., Lewis, P.A., 2014b. Complementary roles of slowwave sleep and rapid eye movement sleep in emotional memory consolidation. Cerebr. Cortex bht349. https://doi.org/10.1093/cercor/bht349.

Carrier, J., Land, S., Buysse, D.J., Kupfer, D.J., Monk, T.H., 2001. The effects of age and gender on sleep EEG power spectral density in the middle years of life (ages 20-60 years old). Psychophysiology 38 (2), 232-242.

Cecere, R., Rees, G., Romei, V., 2015. Individual differences in alpha frequency drive crossmodal illusory perception. Curr. Biol. 25 (2), 231-235.

Cellini, N., Torre, J., Stegagno, L., Sarlo, M., 2016. Sleep before and after learning promotes the consolidation of both neutral and emotional information regardless of REM presence. Neurobiol. Learn. Mem. 133, 136-144.

Chatburn, A., Coussens, S., Lushington, K., Kennedy, D., Baumert, M., Kohler, M., 2013. Sleep spindle activity and cognitive performance in healthy children. Sleep 36 (2), 237-243.

Chatburn, A., Lushington, K., Kohler, M.J., 2014. Complex associative memory processing and sleep: a systematic review and meta-analysis of behavioural evidence and underlying EEG mechanisms. Neurosci. Biobehav. Rev. 47, 646-655.

Conway, A.R., Kane, M.J., Engle, R.W., 2003. Working memory capacity and its relation to general intelligence. Trends Cognit. Sci. 7 (12), 547-552. https://doi.org/ 10.1016/j.tics.2003.10.005.

Corcoran, A.W., Alday, P.M., Schlesewsky, M., Bornkessel-Schlesewsky, I., 2018. Towards a reliable, automated method of individual alpha frequency (IAF) quantification. Psychophysiology. https://doi.org/10.1111/psyp.13064.

Corcoran, A. W., Alday, P. M., Schlesewsky, M., \& Bornkessel-Schlesewsky, I. (2019). restingIAF v1.0.3 [software]. Retrieved from https://github.com/corcorana/resti ngIAF. doi: 10.5281/zenodo.2575868.

Cox, R., Schapiro, A.C., Manoach, D.S., Stickgold, R., 2017. Individual differences in frequency and topography of slow and fast sleep spindles. Front. Hum. Neurosci. 11, 433.

Cross, Z.R., Helfrich, R.F., Kohler, M.J., Corcoran, A.W., Coussens, S., Zou-Williams, L., et al., 2020. Slow wave-spindle coupling during sleep predicts language learning and associated oscillatory activity. BioRxiv.

Crowley, R., Bendor, D., Javadi, A.H., 2019. A review of neurobiological factors underlying the selective enhancement of memory at encoding, consolidation, and retrieval. Prog. Neurobiol. 179, 101615. https://doi.org/10.1016/j. pneurobio.2019.04.004.

Cunningham, T.J., Chambers, A.M., Payne, J.D., 2014. Prospection and emotional memory: how expectation affects emotional memory formation following sleep and wake. Front. Psychol. 5 (862), 1-9. https://doi.org/10.3389/fpsyg.2014.00862.

Delorme, A., Makeig, S., 2004. EEGLAB: an open source toolbox for analysis of singletrial EEG dynamics including independent component analysis. J. Neurosci. Methods 134 (1), 9-21. https://doi.org/10.1016/j.jneumeth.2003.10.009.

Diekelmann, S., Wilhelm, I., Born, J., 2009. The whats and whens of sleep-dependent memory consolidation. Sleep Med. Rev. 13 (5), 308-321. https://doi.org/10.1016/j. smrv 2008.08.002.

Durrant, S.J., Cairney, S.A., McDermott, C., Lewis, P.A., 2015. Schema-conformant memories are preferentially consolidated during REM sleep. Neurobiol. Learn. Mem. 122, 41-50.

Ellenbogen, J.M., Hulbert, J.C., Stickgold, R., Dinges, D.F., Thompson-Schill, S.L., 2006. Interfering with theories of sleep and memory: sleep, declarative memory, and associative interference. Curr. Biol. 16 (13), 1290-1294. https://doi.org/10.1016/j. cub.2006.05.024.

Faul, F., Erdfelder, E., Lang, A.G., Buchner, A., 2007. G*Power 3: a flexible statistical power analysis program for the social, behavioral, and biomedical sciences. Behav. Res. Methods 39 (2), 175-191. https://doi.org/10.3758/BF03193146.

Fenn, K.M., Hambrick, D.Z., 2012. Individual differences in working memory capacity predict sleep-dependent memory consolidation. J. Exp. Psychol. Gen. 141 (3), 404.

Fenn, K.M., Hambrick, D.Z., 2015. General intelligence predicts memory change across sleep. Psychon. Bull. Rev. 22 (3), 791-799.

Fernandez, L.M., Lüthi, A., 2020. Sleep spindles: mechanisms and functions. Physiol. Rev. 100 (2), 805-868.

Fogel, S.M., Smith, C.T., 2011. The function of the sleep spindle: a physiological index of intelligence and a mechanism for sleep-dependent memory consolidation. Neurosci. Biobehav. Rev. 35 (5), 1154-1165.

Fox, J., \& Weisberg, S. (2011). Multivariate Linear Models in R. An R Companion to Applied Regression. (Los Angeles: Thousand Oaks).

Grandy, T.H., Werkle-Bergner, M., Chicherio, C., Lövdén, M., Schmiedek, F., Lindenberger, U., 2013a. Individual alpha peak frequency is related to latent factors of general cognitive abilities. Neuroimage 79, 10-18.

Grandy, T.H., Werkle-Bergner, M., Chicherio, C., Schmiedek, F., Lövdén, M. Lindenberger, U., 2013b. Peak individual alpha frequency qualifies as a stable neurophysiological trait marker in healthy younger and older adults. Psychophysiology 50 (6), 570-582.

Groch, S., McMakin, D., Guggenbühl, P., Rasch, B., Huber, R., Wilhelm, I., 2016. Memory cueing during sleep modifies the interpretation of ambiguous scenes in adolescents and adults. Developmental Cognitive Neuroscience 17, 10-18.

Groch, S., Wilhelm, I., Diekelmann, S., Born, J., 2013. The role of REM sleep in the processing of emotional memories: evidence from behavior and event-related potentials. Neurobiol. Learn. Mem. 99, 1-9. https://doi.org/10.1016/j. nlm.2012.10.006.
Groch, S., Zinke, K., Wilhelm, I., Born, J., 2015. Dissociating the contributions of slow wave sleep and rapid eye movement sleep to emotional item and source memory. Neurobiol. Learn. Mem. 122, 122-130.

Grosmark, A.D., Buzsáki, G., 2016. Diversity in neural firing dynamics supports both rigid and learned hippocampal sequences. Science 351 (6280), 1440-1443.

Hanert, A., Weber, F.D., Pedersen, A., Born, J., Bartsch, T., 2017. Sleep in humans stabilizes pattern separation performance. J. Neurosci. 37 (50), 12238-12246.

Hautus, M.J., 1995. Corrections for extreme proportions and their biasing effects on estimated values of d'. Behav. Res. Methods Instrum. Comput. 27 (1), 46-51.

Heib, D.P., Hoedlmoser, K., Anderer, P., Gruber, G., Zeitlhofer, J., Schabus, M., 2015 Oscillatory theta activity during memory formation and its impact on overnight consolidation: a missing link? J. Cognit. Neurosci. https://doi.org/10.1162/jocn_a 00804.

Helfrich, R.F., Lendner, J.D., Mander, B.A., Guillen, H., Paff, M., Mnatsakanyan, L., et al., 2019. Bidirectional prefrontal-hippocampal dynamics organize information transfer during sleep in humans. Nat. Commun. 10 (1), 1-16.

Helfrich, R.F., Mander, B.A., Jagust, W.J., Knight, R.T., Walker, M.P., 2018. Old brains come uncoupled in sleep: slow wave-spindle synchrony, brain atrophy, and forgetting. Neuron 97 (1), 221-230.

Hobson, J.A., Friston, K.J., 2012. Waking and dreaming consciousness: neurobiological and functional considerations. Prog. Neurobiol. 98 (1), 82-98.

Holm, S., 1979. A simple sequentially rejective multiple test procedure. Scand. J. Stat. 65-70.

Hutchison, I.C., Rathore, S., 2015. The role of REM theta activity in emotional memory. Front. Psychol. 6, 1439. https://doi.org/10.3389/fpsyg.2015.01439.

Inostroza, M., Born, J., 2013. Sleep for preserving and transforming episodic memory. Annu. Rev. Neurosci. 36, 79-102.

Jach, H.K., Feuerriegel, D., Smillie, L.D., 2020. Decoding personality trait measures from resting EEG: an exploratory report. Cortex. https://doi.org/10.1016/j. cortex.2020.05.013.

Jann, K., Koenig, T., Dierks, T., Boesch, C., Federspiel, A., 2010. Association of individual resting state EEG alpha frequency and cerebral blood flow. Neuroimage 51 (1), 365-372. https://doi.org/10.1016/j.neuroimage.2010.02.024.

Judd, C.M., Westfall, J., Kenny, D.A., 2012. Treating stimuli as a random factor in social psychology: a new and comprehensive solution to a pervasive but largely ignored problem. J. Pers. Soc. Psychol. 103 (1), 54. https://doi.org/10.1037/a0028347.

Keenan, E.K., Tiplady, B., Priestley, C.M., Rogers, P.J., 2014. Naturalistic effects of five days of bedtime caffeine use on sleep, next-day cognitive performance, and mood. J. Caffeine Res. 4 (1), 13-20. https://doi.org/10.1089/jcr.2011.0030.

Kim, S.Y., Kark, S.M., Daley, R.T., Alger, S.E., Rebouças, D., Kensinger, E.A., Payne, J.D., 2020. Interactive effects of stress reactivity and rapid eye movement sleep theta activity on emotional memory formation. Hippocampus 30 (8), 829-841.

Klimesch, W., 1999. EEG alpha and theta oscillations reflect cognitive and memory performance: a review and analysis. Brain Res. Rev. 29 (2), 169-195. https://doi. org/10.1016/S0165-0173(98)00056-3.

Klimesch, W., 2012. Alpha-band oscillations, attention, and controlled access to stored information. Trends in Cognitive Neuroscience 16 (12), 606-617. https://doi.org/ 10.1016/j.tics.2012.10.007.

Klimesch, W., 2018. The frequency architecture of brain and brain body oscillations: an analysis. Eur. J. Neurosci. 48 (7), 2431-2453.

Klimesch, W., Doppelmayr, M., Hanslmayr, S., 2006. Upper alpha ERD and absolute power: their meaning for memory performance. Prog. Brain Res. 159, 151-165.

Klimesch, W., Schimke, H., Pfurtscheller, G., 1993. Alpha frequency, cognitive load and memory performance. Brain Topogr. 5 (3), 241-251.

Klimesch, W., Schimke, H., Doppelmayr, M., Ripper, B., Schwaiger, J., Pfurtscheller, G., 1996. Event-related desynchronization (ERD) and the Dm effect: does alpha desynchronization during encoding predict later recall performance? Int. J. Psychophysiol. 24 (1-2), 47-60.

Klinzing, J.G., Rasch, B., Born, J., Diekelmann, S., 2016. Sleep's role in the reconsolidation of declarative memories. Neurobiol. Learn. Mem. 136, 166-173.

Lang, P. J., Bradley, M. M., \& Cuthbert, B. N. (2008). reportInternational Affective Picture System (IAPS): Affective Ratings of Pictures and Instruction Manual. Technical Report A-8. University of Florida, Gainesville, FL.

Lang, P., Bradley, M.M., 2007. The international affective picture System (IAPS) in the study of emotion and attention. Handbook of emotion elicitation and assessment 29.

Lechinger, J., Heib, D.P.J., Gruber, W., Schabus, M., Klimesch, W., 2015. Heartbeatrelated EEG amplitude and phase modulations from wakefulness to deep sleep: interactions with sleep spindles and slow oscillations. Psychophysiology 52 (11), 1441-1450.

LeDoux, J., 2017. The amygdala. Curr. Biol. 17, 868-874.

Lehmann, M., Seifritz, E., Rasch, B., 2016. Sleep benefits emotional and neutral associative memories equally. Somnologie 20 (1), 47-53.

Lewis, P.A., Knoblich, G., Poe, G., 2018. How memory replay in sleep boosts creative problem-solving. Trends Cognit. Sci. 22 (6), 491-503.

Lipinska, G., Stuart, B., Thomas, K.G., Baldwin, D.S., Bolinger, E., 2019. Preferential consolidation of emotional memory during sleep: a meta-analysis. Front. Psychol. $10,1014$.

Lopez-Calderon, J., Luck, S.J., 2014. ERPLAB: an open-source toolbox for the analysis of event-related potentials. Front. Hum. Neurosci. 8, 213.

Lutz, N.D., Wolf, I., Hübner, S., Born, J., Rauss, K., 2018. Sleep strengthens predictive sequence coding. J. Neurosci. 38 (42), 8989-9000.

MacGregor-Fors, I., Payton, M.E., 2013. Contrasting diversity values: statistical inferences based on overlapping confidence intervals. PloS One 8 (2).

Mathôt, S., Schreij, D., Theeuwes, J., 2012. OpenSesame: an open-source, graphical experiment builder for the social sciences. Behav. Res. Methods 44 (2), 314-324. https://doi.org/10.3758/s13428-011-0168-7. 
McGaugh, J.L., 2004. The amygdala modulates the consolidation of memories of emotionally arousing experiences. Annu. Rev. Neurosci. 27, 1-28.

Mölle, M., Marshall, L., Gais, S., Born, J., 2002. Grouping of spindle activity during slow oscillations in human non-rapid eye movement sleep. J. Neurosci. 22 (24), 10941-10947.

Morgenthaler, J., Wiesner, C.D., Hinze, K., Abels, L.C., Prehn-Kristensen, A., Göder, R., 2014. Selective REM-sleep deprivation does not diminish emotional memory consolidation in young healthy subjects. PloS One 9 (2). https://doi.org/10.1371/ journal.pone.0089849.

Murty, V.P., Ritchey, M., Adcock, R.A., LaBar, K.S., 2010. fMRI studies of successful emotional memory encoding: a quantitative meta-analysis. Neuropsychologia 48 (12), 3459-3469. https://doi.org/10.1016/j.neuropsychologia.2010.07.030.

Ngo, H.V.V., Martinetz, T., Born, J., Mölle, M., 2013. Auditory closed-loop stimulation of the sleep slow oscillation enhances memory. Neuron 78 (3), 545-553.

Nishida, M., Pearsall, J., Buckner, R.L., Walker, M.P., 2009. REM sleep, prefrontal theta, and the consolidation of human emotional memory. Cerebr. Cortex 19 (5), 1158-1166. https://doi.org/10.1093/cercor/bhn155.

Oostenveld, R., Fries, P., Maris, E., \& Schoffelen, J. M. (2011). FieldTrip: Open Source Software for Advanced Analysis of MEG, EEG, and Invasive Electrophysiological Data. Computational intelligence and neuroscience, 2011.

Payne, J.D., Kensinger, E.A., Wamsley, E.J., Spreng, R.N., Alger, S.E., Gibler, K., et al., 2015. Napping and the selective consolidation of negative aspects of scenes. Emotion 15 (2), 176. https://doi.org/10.1037/a0038683.

Payne, J.D., Stickgold, R., Swanberg, K., Kensinger, E.A., 2008. Sleep preferentially enhances memory for emotional components of scenes. Psychol. Sci. 19 (8), 781-788. https://doi.org/10.1111/j.1467-9280.2008.02157.x.

Payne, J., Chambers, A.M., Kensinger, E.A., 2012. Sleep promotes lasting changes in selective memory for emotional scenes. Front. Integr. Neurosci. 6 https://doi.org/ 10.3389/fnint.2012.00108.

Piosczyk, H., Holz, J., Feige, B., Spiegelhalder, K., Weber, F., Landmann, N., et al., 2013. The effect of sleep-specific brain activity versus reduced stimulus interference on declarative memory consolidation. J. Sleep Res. 22 (4), 406-413.

Posthuma, D., Neale, M.C., Boomsma, D.I., De Geus, E.J.C., 2001. Are smarter brains running faster? Heritability of alpha peak frequency, IQ, and their interrelation. Behav. Genet. 31 (6), 567-579.

Prehn-Kristensen, A., Munz, M., Molzow, I., Wilhelm, I., Wiesner, C.D., Baving, L., 2013 Sleep promotes consolidation of emotional memory in healthy children but not in children with attention-deficit hyperactivity disorder. PloS One 8 (5), e65098. https://doi.org/10.1371/journal.pone.0065098.

Rasch, B., Born, J., 2013. About sleep's role in memory. Physiol. Rev. 93 (2), 681-786. https://doi.org/10.1152/physrev.00032.2012.

Saletin, J.M., Walker, M.P., 2012. Nocturnal mnemonics: sleep and hippocampal memory processing. Front. Neurol. 3, 59. https://doi.org/10.3389/ fneur.2012.00059.

Samaha, J., Postle, B.R., 2015. The speed of alpha-band oscillations predicts the temporal resolution of visual perception. Curr. Biol. 25 (22), 2985-2990.

Sassenhagen, J., Alday, P.M., 2016. A common misapplication of statistical inference: nuisance control with null-hypothesis significance tests. Brain Lang. 162, 42-45.

Schabus, M., Gruber, G., Parapatics, S., Sauter, C., Klösch, G., Anderer, P., et al., 2004. Sleep spindles and their significance for declarative memory consolidation. Sleep 27 (8), 1479-1485.
Schabus, M., Hoedlmoser, K., Pecherstorfer, T., Anderer, P., Gruber, G., Parapatics, S., et al., 2008. Interindividual sleep spindle differences and their relation to learningrelated enhancements. Brain Res. 1191, 127-135.

Stanislaw, H., Todorov, N., 1999. Calculation of signal detection theory measures. Behav. Res. Methods Instrum. Comput. 31 (1), 137-149.

Staresina, B.P., Bergmann, T.O., Bonnefond, M., van der Meij, R., Jensen, O., Deuker, L., et al., 2015. Hierarchical nesting of slow oscillations, spindles and ripples in the human hippocampus during sleep. Nat. Neurosci. 18, 1679-1686. https://doi.org/ 10.1038/nn.4119.

Sterpenich, V., Albouy, G., Darsaud, A., Schmidt, C., Vandewalle, G., Vu, T.T.D., Collette, F., 2009. Sleep promotes the neural reorganization of remote emotional memory. J. Neurosci. 29 (16), 5143-5152.

Studte, S., Bridger, E., Mecklinger, A., 2017. Sleep spindles during a nap correlate with post sleep memory performance for highly rewarded word-pairs. Brain Lang. 167, 28-35.

Szucs, D., Ioannidis, J., 2017. When null hypothesis significance testing is unsuitable for research: a reassessment. Front. Hum. Neurosci. 11, 390. https://doi.org/10.3389/ fnhum.2017.00390.

Talmi, D., 2013. Enhanced emotional memory: cognitive and neural mechanisms. Curr. Dir. Psychol. Sci. 22 (6), 430-436.

Tassi, P., Muzet, A., 2000. Sleep inertia. Sleep Med. Rev. 4 (4), 341-353. https://doi.org/ 10.1053/smrv.2000.0098.

Tononi, G., Cirelli, C., 2014. Sleep and the price of plasticity: from synaptic and cellular homeostasis to memory consolidation and integration. Neuron 81 (1), 12-34. https://doi.org/10.1016/j.neuron.2013.12.025.

Tufte, E. R. (1983). The Visual Display of Quantitative Information Graphics Press. Cheshire, Connecticut.

Ujma, P.P., 2018. Sleep spindles and general cognitive ability-A meta-analysis. Sleep Spindles \& Cortical Up States 1-17.

van den Elzen, S., \& van Wijk, J. J. (2013, June). Small multiples, large singles: a new approach for visual data exploration. In Computer Graphics Forum (Vol. vol. 32, No. 3pt2, pp. 191-200). Oxford, UK: Blackwell Publishing Ltd.

Van Dongen, H.P., Olofsen, E., Dinges, D.F., Maislin, G., 2004. Mixed-model regression analysis and dealing with interindividual differences. Methods Enzymol. 384, 139-171.

van Schalkwijk, F.J., Sauter, C., Hoedlmoser, K., Heib, D.P., Klösch, G., Moser, D., et al., 2019. The effect of daytime napping and full-night sleep on the consolidation of declarative and procedural information. J. Sleep Res. 28 (1), e12649.

Vuilleumier, P., 2005. How brains beware: neural mechanisms of emotional attention. Trends Cognit. Sci. 9 (12), 585-594.

Wagner, U., Gais, S., Born, J., 2001. Emotional memory formation is enhanced across sleep intervals with high amounts of rapid eye movement sleep. Learn. Mem. 8 (2), 112-119. https://doi.org/10.1101/1m.36801.

Walker, M.P., 2010. Sleep, memory and emotion. Prog. Brain Res. 185, 49-68.

Wickham, H. (2009). ggplot2: Elegant Graphics for Data Analysis Springer-Verlag. New York.

Wislowska, M., Heib, D.P., Griessenberger, H., Hoedlmoser, K., Schabus, M., 2017. Individual baseline memory performance and its significance for sleep-dependent memory consolidation. Sleep Spindles \& Cortical Up States 1 (1), 2-13. 